\title{
Neuronal Organization in the Inferior Colliculus Revisited with Cell-Type-Dependent Monosynaptic Tracing
}

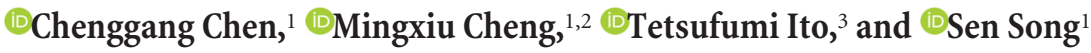 \\ ${ }^{1}$ Tsinghua Laboratory of Brain and Intelligence and Department of Biomedical Engineering, Beijing Innovation Center for Future Chip, Center for Brain- \\ Inspired Computing Research, McGovern Institute for Brain Research, Tsinghua University, Beijing, 100084, China, ${ }^{2}$ National Institute of Biological \\ Sciences, Beijing, 102206, China, and ${ }^{3}$ Anatomy II, School of Medicine, Kanazawa Medical University, Uchinada, Ishikawa, 920-0293, Japan
}

The inferior colliculus (IC) is a critical integration center in the auditory pathway. However, because the inputs to the IC have typically been studied by the use of conventional anterograde and retrograde tracers, the neuronal organization and cell-type-specific connections in the IC are poorly understood. Here, we used monosynaptic rabies tracing and in situ hybridization combined with excitatory and inhibitory Cre transgenic mouse lines of both sexes to characterize the brainwide and cell-type-specific inputs to specific neuron types within the lemniscal IC core and nonlemniscal IC shell. We observed that both excitatory and inhibitory neurons of the IC shell predominantly received ascending inputs rather than descending or core inputs. Correlation and clustering analyses revealed two groups of excitatory neurons in the shell: one received inputs from a combination of ascending nuclei, and the other received inputs from a combination of descending nuclei, neuromodulatory nuclei, and the contralateral IC. In contrast, inhibitory neurons in the core received inputs from the same combination of all nuclei. After normalizing the extrinsic inputs, we found that core inhibitory neurons received a higher proportion of inhibitory inputs from the ventral nucleus of the lateral lemniscus than excitatory neurons. Furthermore, the inhibitory neurons preferentially received inhibitory inputs from the contralateral IC shell. Because IC inhibitory neurons innervate the thalamus and contralateral IC, the inhibitory inputs we uncovered here suggest two long-range disinhibitory circuits. In summary, we found: (1) dominant ascending inputs to the shell, (2) two subpopulations of shell excitatory neurons, and (3) two disinhibitory circuits.

Key words: in situ hybridization; inferior colliculus; rabies virus; transgenic mice

Significance Statement

Sound undergoes extensive processing in the brainstem. The inferior colliculus (IC) core is classically viewed as the integration center for ascending auditory information, whereas the IC shell integrates descending feedback information. Here, we demonstrate that ascending inputs predominated in the IC shell but appeared to be separated from the descending inputs. The presence of inhibitory projection neurons is a unique feature of the auditory ascending pathways, but the connections of these neurons are poorly understood. Interestingly, we also found that inhibitory neurons in the IC core and shell preferentially received inhibitory inputs from ascending nuclei and contralateral IC, respectively. Therefore, our results suggest a bipartite domain in the IC shell and disinhibitory circuits in the IC.

\section{Introduction}

The auditory midbrain or inferior colliculus (IC) is a critical integration center of the auditory pathway and is divided into

Received July 31, 2017; revised Feb. 2, 2018; accepted Feb. 7, 2018.

Author contributions: C.C. wrote the first draft of the paper; C.C., T.I., and S.S. edited the paper; C.C., T.I., and S.S. designed research; C.C. and M.C. performed research; C.C. and T.I. analyzed data; C.C., T.I., and S.S. wrote the paper.

This work was supported by National Natural Science Foundation of China 31571095 and 91332122 to S.S., Special Fund of Suzhou-Tsinghua Innovation Leading Action to S.S., Beijing Program on the Study of Brain-Inspired Computing System and Related Core Technologies to S.S., Beijing Innovation Center for Future Chip to S.S., Chinese Academy of Sciences Institute of Psychology Key Laboratory of Mental Health Open Research Grant KLMH2012K02 to S.S., Ministry of Education, Science, and Culture of Japan KAKENHI Grants 16 K07026 and 16H01501 to T.I., and Takahashi Industrial and Economic Research Foundation to T.I. We thank D. Cai for technical assistance; and Li Shen, Kexin Yuan, Xiaoqin Wang, and Douglas Oliver for comments on the manuscript.

The authors declare no competing financial interests. core (central nucleus) and shell (dorsal and lateral nucleus) subdivisions according to dendritic morphology and axonal trajectories (Oliver and Morest, 1984). The IC core primarily receives ascending brainstem inputs (Cant and Benson, 2006). In contrast, physiological and behavioral studies have demonstrated that the IC shell not only receives ascending inputs (Loftus et al., 2008) but also descending auditory cortex (AC) and IC core inputs (Winer et al., 1998; Winer and Schreiner, 2005). IC shell

Correspondence should be addressed to either of the following: Dr. Sen Song, Tsinghua University, Beijing 100084, China, E-mail: songsen@tsinghua.edu.cn; or Dr. Tetsufumi Ito, Kanazawa Medical University, Uchinada, Ishikawa, 920-0293, Japan, E-mail: itot@kanazawa-med.ac.jp.

DOI:10.1523/JNEUROSCI.2173-17.2018

Copyright $\odot 2018$ the authors $\quad 0270-6474 / 18 / 383318-15 \$ 15.00 / 0$ 
neurons display broader tuning (Syka et al., 2000) and stronger stimulus-specific adaptation (SSA) (Shen et al., 2015) than core neurons, suggesting an influence of both descending and core inputs. On the other hand, the shell neurons also exhibit short response latencies similar to the IC core neurons (Syka et al., 2000), and shell stimulation directly induces innate auditory behavior (Xiong et al., 2015), implying an effect of brainstem inputs. However, whether the IC shell primarily receives descending and core inputs or ascending inputs is still unclear.

The core and shell subdivisions can also be divided into finer units of IC neuronal organization (i.e., synaptic domains), which receive inputs from different combinations of nuclei and are considered to be a fundamental feature of the IC and superior colliculus (SC) (Oliver, 2005). Synaptic domains or functional modules exist in specific locations of the core and shell, exhibit specific monaural, binaural, and SSA responses, and receive specific ascending or descending inputs (Loftus et al., 2010; Ayala et al., 2015). However, previous studies only defined the synaptic domains by anterograde or retrograde tracing in terms of their anatomical location and not on a neuronal level. When neurons, such as neurons in the deep shell layer (Malmierca et al., 2011) or stellate neurons in the core (Oliver, 2005), project dendrites out of a specific location (i.e., injection site), the pattern and number of inputs received by these neurons may be severely underestimated.

In addition to anatomical subdivisions of the core, shell, and finer synaptic domains, IC neurons can be subdivided into two major classes: excitatory glutamatergic neurons and inhibitory GABAergic neurons (Ito et al., 2011). Twenty-five percent of IC neurons are GABAergic (Merchán et al., 2005). One distinctive feature of the rat IC is that $40 \%$ of the thalamic projection neurons are GABAergic (Peruzzi et al., 1997). GABAergic projection neurons are characterized by large somata that are densely innervated by vesicular glutamate transporter 2 (Vglut2)-positive axosomatic terminals (Ito et al., 2009, 2015; Ito and Oliver, 2014). Furthermore, the large GABAergic projection neurons receive shorter latency excitatory inputs than other IC neurons (Geis and Borst, 2013). These findings strongly suggest that the organization of inputs to GABAergic projection neurons differs substantially from that of glutamatergic neurons. Although both excitatory and inhibitory inputs to the IC have been observed (González-Hernández et al., 1996; Patel et al., 2017), the cell types of their postsynaptic targets remain mostly unknown. One study that combined electron microscopy, anterograde tracing, and immunocytochemistry showed preliminary evidence that the majority of terminals from the AC contacted non-GABAergic neurons (Nakamoto et al., 2013a). However, this study did not provide the proportion of total inputs to each cell type, making it difficult to assess their contribution to the auditory midbrain circuitry.

Here, we used the monosynaptic rabies tracing technique (Wickersham et al., 2007; Watabe-Uchida et al., 2012; Weissbourd et al., 2014; Menegas et al., 2015; Do et al., 2016) combined with excitatory and inhibitory Cre driver lines to characterize the brainwide inputs to specified IC neuron types within specific subdivisions. Furthermore, combining the viral tracing with in situ hybridization (ISH) histochemistry, we clarified the cell types that give rise to the commissural inputs on specific cell types, allowing us to reveal parallel ascending pathways in the IC core and shell, different combinations of inputs to excitatory neurons, and two long-range disinhibitory connections in the IC.

\section{Materials and Methods}

Animal surgery and virus injection. All experiments were approved and conducted in accordance with the guidelines set by the Tsinghua University Animal Care and Use Committee. For all experiments, 27 Vglut2-Cre (JAX: 016963) and 25 VGAT-Cre (JAX: 016962) and 8 C57BL/6 wild-type (Vital River Laboratory) adult (2-5 month) mice of both sexes were used.

The mice were anesthetized with pentobarbital (40 mg/kg, i.p.), and eye ointment was used to prevent dry eyes during the surgery. The head skin was washed by alternating application of Betadine and $70 \%(\mathrm{v} / \mathrm{v})$ alcohol three times to prevent inflammation. The skin over the IC was cut with sterile scissors and forceps, after which the animals were mounted in a stereotaxic holder, and a portion of the occipital bone $\sim 1 \mathrm{~mm}$ in diameter was thinned with a $0.5 \mathrm{~mm}$ drill bit. When the boundary of the IC could be clearly defined by visualization of the transverse sinus, cerebellum, and SC, the drilling stopped, and a 26-gauge needle was used to remove the thinned skull ( $\sim 50 \mu \mathrm{m}$ in diameter) at the desired location to allow for virus injection. The virus injections sites along the mediolateral and dorsoventral planes were guided using stereotaxic coordinates: central nucleus of the IC (ICC): $1000 \mu \mathrm{m}$ lateral from midline, 800-1200 $\mu \mathrm{m}$ deep from brain surface; dorsal nucleus of the IC (ICD): $300 \mu \mathrm{m}$ lateral, 100-300 $\mu \mathrm{m}$ deep; and external nucleus of the IC (ICX): $1500 \mu \mathrm{m}$ lateral, 300-500 $\mu \mathrm{m}$ deep). The injection was made $200 \mu \mathrm{m}$ caudal to the transverse sinus to avoid blood vessel rupture.

A microsyringe pump (Micro4, WPI) was used to inject $100 \mathrm{nl}$ of a viral mixture of AAV9 helper viruses, rAAV-EF1 $\alpha$-DIO-His-EGFP-2aTVA-WPRE-pA and rAAV-EF1 $\alpha$-DIO-RG-WPRE-pA (BrainVTA), which express avian-specific retroviral receptor (TVA), GFP, and rabies glycoprotein $(\mathrm{RG})$ driven by the elongation factor $1 \alpha(\mathrm{EF} 1 \alpha)$ promoter. The concentration of the viruses was $2 \times 10^{12}$ vector genomes (vg) per milliliter. Two weeks after the injection of the AAV helper viruses, $50 \mathrm{nl}$ $\mathrm{SAD} \Delta \mathrm{G}$-DsRed rabies virus (RV) at $2 \times 10^{8} \mathrm{vg} / \mathrm{ml}$ (BrainVTA) was injected into the same location using a similar procedure. For the control experiments mentioned in Figure $1 E, F$, we only injected rAAV-EF1 $\alpha$ DIO-RG-WPRE-pA and SAD $\Delta$ G-DsRed RV, or rAAV-EF1 $\alpha$-DIO-HisEGFP-2a-TVA-WPRE-pA and SAD $\Delta$ G-DsRed RV, respectively. For the control experiments mentioned in Figure $1 G-K$, we injected rAAVEF1 $\alpha$-DIO-His-EGFP-2a-TVA-WPRE-pA, rAAV-EF1 $\alpha$-DIO-RG-WPRE$\mathrm{pA}$, and SAD $\Delta \mathrm{G}$-DsRed RV to the C57BL/6 wild-type mice. The volume and titer of AAV and RV used in the control experiments were the same as regular experiments.

Histology and ISH. One week after the injection of RV, the mice were anesthetized with pentobarbital $(200 \mathrm{mg} / \mathrm{kg}$, i.p.) and transcardially perfused with saline followed by $4 \%$ PFA in PBS. After postfixation with the same fixative overnight, the brains were cryoprotected by immersion in $20 \%$ and $30 \%$ sucrose in $0.1 \mathrm{M} \mathrm{PB}$ for 12 and $24 \mathrm{~h}$, respectively. The brains were then embedded in OCT compound (Tissue-Tek, Sakura Finetek) and sliced using a cryostat (CM1950, Leica Biosystems) at a thickness of $50 \mu \mathrm{m}$. We examined the brain sections from the caudal end of the cochlear nuclei $(\mathrm{CN})$ ( $6.48 \mathrm{~mm}$ caudal from bregma) to the subparafascicular thalamic nucleus (SPF) (1.70 mm caudal from bregma). Every other section was collected and mounted on gelatin-coated slides. For DAPI staining, the brain sections mounted on slides were washed with PBS for $10 \mathrm{~min}$, followed by the addition of 1: 2000 DAPI (D1306, Thermo Fisher Scientific). Five minutes later, the sections were washed again for another $10 \mathrm{~min}$ with PBS. For Nissl staining, the brain sections were collected in a 6-well cell culture plate, rinsed with PBS for half an hour, and then washed with PBS plus $0.1 \%$ Triton X-100 for $10 \mathrm{~min}$ and PBS for 5 min. Later, 1:200 fluorescent Nissl (N21479, Thermo Fisher Scientific) was added to the buffer, and the sections were incubated at room temperature for $20 \mathrm{~min}$. Finally, the sections were washed with PBS overnight at $4^{\circ} \mathrm{C}$.

For the ISH experiments, we prepared Vglut2 and VGAT antisense riboprobes (Table 1) by including a t7 promoter in the $5^{\prime}$-overhang of the forward primer. DNA fragments of the target gene were then obtained from mouse whole-brain cDNA with PCR. Afterward, the DNA product was transcribed with DIG-RNA Labeling Mix (11277073910, Roche) to make the Vglut2 and VGAT probes. 
Table 1. Riboprobes for ISH used in this study

\begin{tabular}{llll}
\hline Probe & Accession no. & Probe region & Primer sequence \\
\hline Vglut2 & NM_080853-3 & $720-1635$ & $\begin{array}{l}\text { Forward: TGGATGGTCGTCAGTATTT } \\
\text { Reverse: ACCGTAAGATTTGGTGT }\end{array}$ \\
VGAT & NM_031782 & 239-1192 & $\begin{array}{l}\text { Forward: AGGGAGACATTCATTATCAGCG } \\
\text { Reverse: AGGGCAACGGGTAGGACA }\end{array}$ \\
\hline
\end{tabular}

Samples were prepared as described above, except that DEPC-treated buffers were used to prevent the degradation of RNA during the perfusion and cryoprotection procedure. DEPC-PTw (DEPC-PBS with $0.1 \%$ Tween 20 ) and $0.5 \%$ Triton $2 \times$ saline sodium citrate (SSC) were used to permeabilize the tissue. Then, the sections were incubated with acetylation buffer, washed with DEPC-PBS three times, and then incubated with the prehybridization buffer ( $50 \%$ formamide, $5 \times$ SSC, 5 mM EDTA, pH $8.0,0.1 \%$ Tween $20,1 \%$ CHAPS) for $2 \mathrm{~h}$. The probe was then diluted in hybridization solution (50\% formamide, $5 \times$ SSC, 5 mM EDTA, pH 8.0, $0.1 \%$ Tween $20,1 \%$ CHAPS, $300 \mu \mathrm{g} / \mathrm{ml}$ tRNA, $1 \times$ Denhardt's solution, $1 \%$ heparin) to $1 \mu \mathrm{g} / \mathrm{ml}$ and incubated with brain sections for $20 \mathrm{~h}$ at $65^{\circ} \mathrm{C}$. After hybridization, sections were incubated with prehybridization buffer at $65^{\circ} \mathrm{C}$ for $30 \mathrm{~min}$, and the same operation was performed in a mixture of TBST and prehybridization buffer at a ratio of 1:1. The sections were then washed with TBST twice, rinsed with TBST and TAE buffer (1:1) once, and washed with TAE buffer three times. Electrophoresis was used to remove the foreign material from the sections. After this, the sections were incubated with anti-digoxigenin-POD (10520200, 1:500, Roche) at $4^{\circ} \mathrm{C}$ for $30 \mathrm{~h}$ and washed with TNT. The TSA Plus Cyanine 5 system (NEL745B001KT, 1:100, PerkinElmer) was then used to detect the primary antibody. The samples were incubated with antiEYFP (511201, rabbit anti-mouse, 1:200, Zen Bioscience) or anti-DsRed (632496, rabbit anti-mouse, 1:250, Clontech) antibodies overnight at $4^{\circ} \mathrm{C}$, so that the fluorescence that had been quenched by the high temperature during hybridization could be measured. Finally, sections were incubated with the AlexaFluor-594-conjugated (A11037, goat antirabbit, 1:500, Thermo Fisher Scientific) secondary antibody, and fluorescence images were obtained with a confocal microscope (A1, Nikon).

Experimental design and statistical analysis. In this study, we compared the inputs between excitatory and inhibitory neurons and between the IC core and shell. For this reason, two variables needed to be controlled.

One variable was the location and size of the injection. The combination of inputs is known to be different not only between the IC core and shell but also between the dorsal and ventral part of the IC core, the superficial and deep layers of the IC shell, and even small synaptic domains within the IC core and shell (Oliver, 2005). To eliminate this sampling bias, we attempted to cover a large area of the IC core and shell with our viral injection. The side effect for this injection strategy is that some virus may have leaked into other subdivisions (see Fig. 2B). For example, when the virus was injected into the dorsal part of the IC core, some neurons infected with the initial tracing virus (i.e., starter neurons) were located in the boundaries of the ICC, ICD, and ICX; conversely, when the injection was located in a deeper layer of the IC shell, the virus leaked, infecting some starter neurons in the IC core. Therefore, we counted the number of starter neurons within the ICC, ICD, ICX, rostral cortex of the IC (RC), and brachial nucleus of the IC (BIC). Then, we classified the injections as located in either the core or shell when the proportion of starter neurons in the IC core or shell was $>70 \%$, respectively. There were some cases in which the combined proportion in the core and shell was $<100 \%$ because a few starter neurons were located in the periaqueductal gray (PAG). In those cases, the inputs from the neuromodulatory nuclei were excluded from the analyses.

The other variable that needed to be controlled was the expression level of the virally introduced molecules. Unlike the AAV helper virus, the titer of the RV decreases considerably within $48 \mathrm{~h}$ of thawing from the $-80^{\circ} \mathrm{C}$ environment. Therefore, to maintain the inoculation efficiency after the RV was thawed, 8 or 12 mice received the RV injection within $1 \mathrm{~d}$, typically within $\sim 6$ or $9 \mathrm{~h}$ of being thawed (requiring $\sim 45 \mathrm{~min}$ for each mouse). During the virus injection, surgeries on the different mouse lines and injection sites were interleaved (i.e., core-Vglut2-Cre, core-
VGAT-Cre, shell-Vglut2-Cre, and shell-VGAT-Cre). For fixation, the animals were transcardially perfused in the same order as the viral injection surgeries (i.e., the core-Vglut2-Cre mice were perfused first).

In the brain regions without starter neurons (i.e., outside of the injected IC), input neurons, which expressed DsRed but not EGFP, were manually counted with a fluorescence microscope (BX51, Olympus) if the labeled cells were sparsely distributed in an isolated nucleus. For the brain regions with complex cytoarchitecture (e.g., superior olivary complex [SOC]) and/or those that had a high-density of labeled cells, images were acquired with a CCD camera (QIClick, QImaging), and the input neurons were counted with ImageJ software (http://imagej.nih.gov/ij/). In several cases, whole-brain sections were imaged with an automated slice scanner (Axio Scan Z1, Carl Zeiss), and the general borders of the nuclei that contained input neurons were determined and drawn using Neurolucida software (MBF Bioscience).

Tiled images of the brain sections with starter neurons, which expressed both DsRed and EGFP, were acquired with a $10 \times$ objective $(\mathrm{NA}=0.45)$ and a confocal laser scanning microscope (A1, Nikon). DsRed and EGFP were excited sequentially with 561 and $488 \mathrm{~nm}$ lasers, and emitted fluorescence was filtered with 570-620 and 500-550 nm bandpass filters, respectively. The laser intensity was adjusted to eliminate the overexposed neurons, as those saturated spots biased the colocalization results (i.e., the starter neurons). The distribution of starter neurons was analyzed with Neurolucida. The cell count of starter neurons was analyzed with Imaris (Bitplane).

We identified the borders of nuclei using the ISH, Nissl counterstain (with the fluorescent NeuroTrace, instead of the cresyl violet and thionin, which would quench the signals of the fluorescent proteins) and brain atlas unless otherwise stated. The borders were defined as follows: The dorsal cochlear nucleus (DCN) and posterior ventral cochlear nucleus (VCN) are separated by the granule cell layer. The anterior VCN is located anterior to the eighth nerve root. Identification of the borders of subnuclei of the SOC was in accordance with Ito et al. (2011). To identify the nuclear borders of the ventral, intermediate, and dorsal nucleus of the lateral lemniscus (VLL/ILL/DLL), the distribution of cells that expressed Vglut2/VGAT mRNA was used, as the VLL and DLL are characterized by numerous VGAT-expressing cells and few Vglut2-expressing cells, whereas the ILL is characterized by numerous Vglut2-expressing cells and few VGAT-expressing cells (Ito et al., 2011). The DLL and IC are separated by Probst's commissure, which connects the bilateral DLL. To identify the nuclear borders of the descending (the AC and the peripeduncular and posterior intralaminar thalamic nuclei, PP, and PIL, respectively) and neuromodulatory nuclei (locus ceruleus; laterodorsal and pedunculopontine tegmental nuclei, LDTg and PPTg; dorsal, median, and rostral linear nuclei of the raphe; SPF), we primarily referenced the Allen Brain Atlas (Lein et al., 2007) (www.brain-map.org) with further consultation of the standard Mouse Brain Atlas (Paxinos and Franklin, 2001).

The IC subdivisions were delineated by the Nissl staining patterns in each subdivision: In the ICC, flat cell bodies are aligned parallel to the isofrequency laminae. In the ICD, although there are still flat cell bodies, their percentage is lower than the ICC. In the ICX, the Nissl stain clearly visualizes three laminae that are almost oblique or perpendicular to the ICC laminae. Furthermore, the Nissl counterstain method has been widely used by previous studies to determine the nuclear borders of the IC (Loftus et al., 2008; Lee and Sherman, 2010; Ito et al., 2011, 2015; Patel et al., 2017). Because dendritic arbors and axon trajectories have been used to characterize the subdivisions of the IC (Oliver and Morest, 1984; Malmierca et al., 1993, 2011), we also referred to the dendritic and axonal morphologies of the DsRed/EGFP-labeled neurons to facilitate the border determination (see Fig. $1 D$, left) (Ito and Oliver, 2014).

To compare the number of inputs between extrinsic sources, we first normalized the number of input neurons in each extrinsic nucleus to the corresponding number of starter neurons and calculated Pearson's correlation coefficients between all pairs of input nuclei with MATLAB (The MathWorks) using the built-in corrcoef function (with a matrix in which the rows were cases and the columns were input nuclei), thereby obtaining a matrix of pairwise Pearson's correlation coefficients. This 


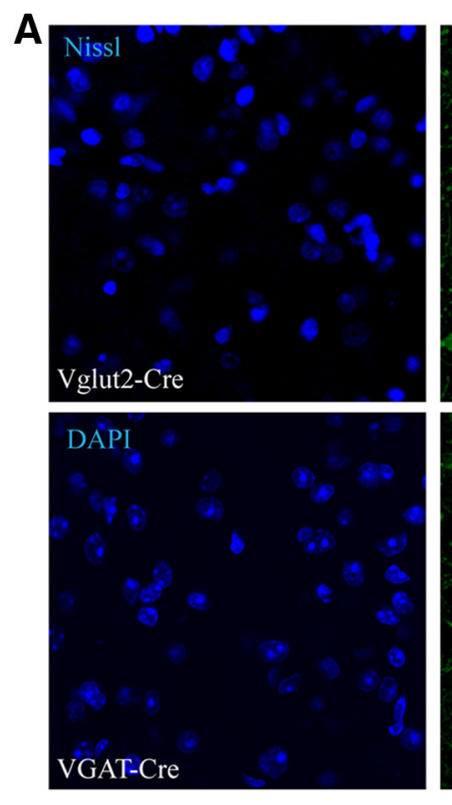

B

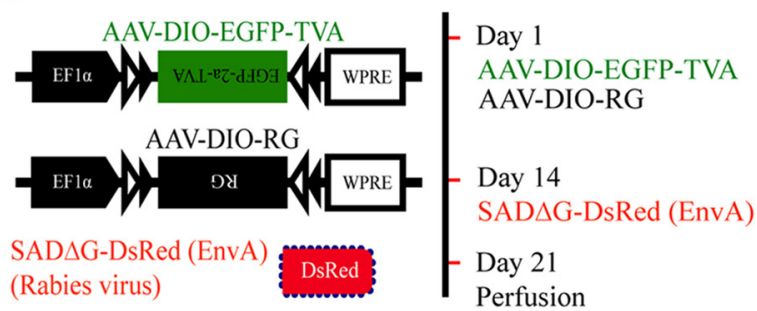

C

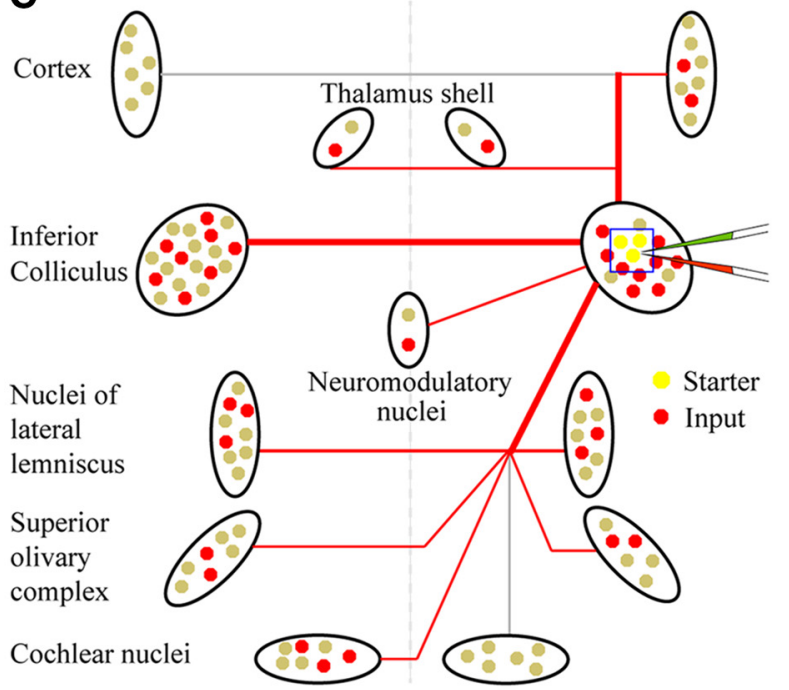

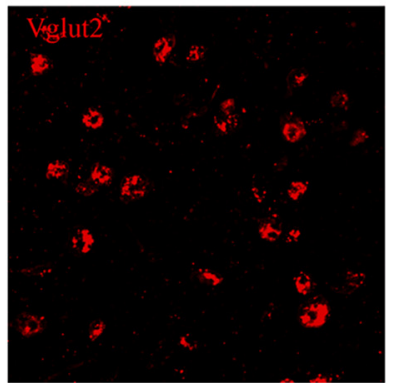
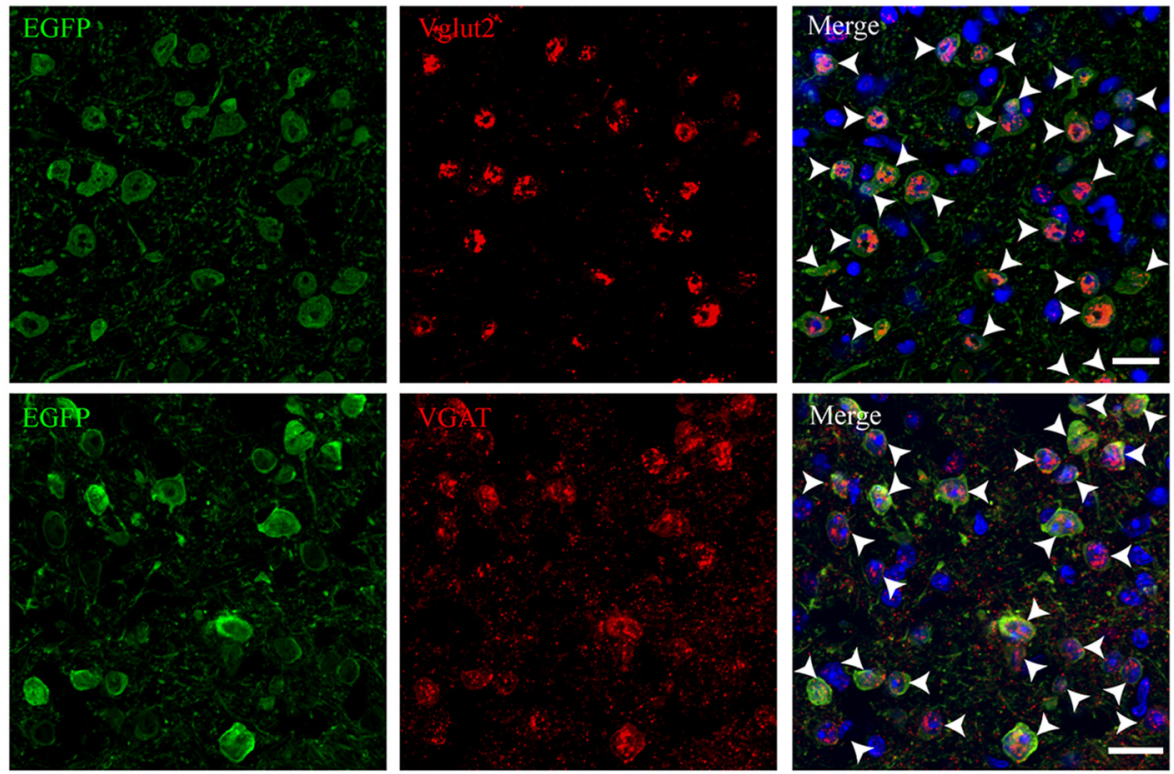

\section{D}
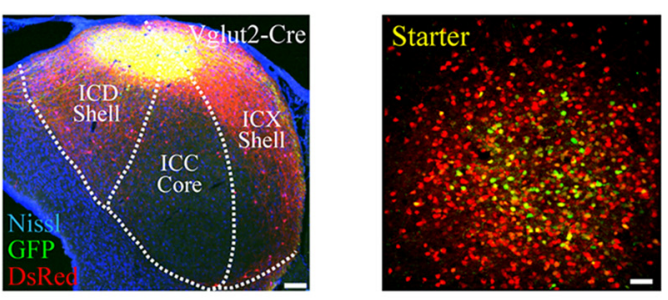

E

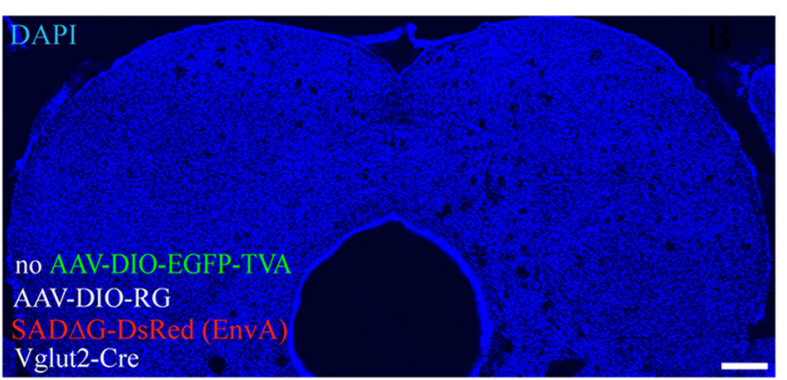

F
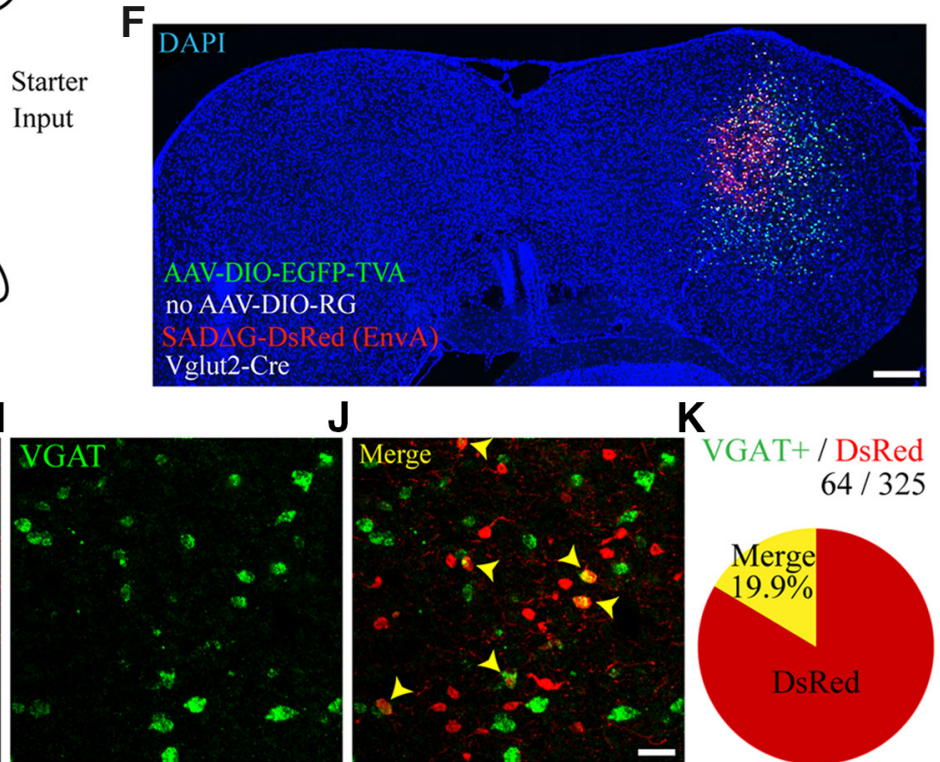

K

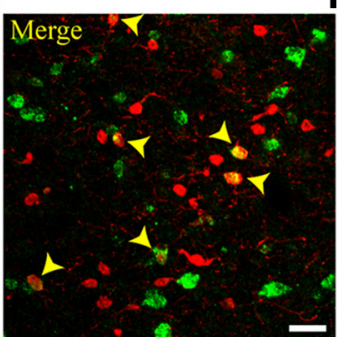

VGAT+/ DsRed

$64 / 325$

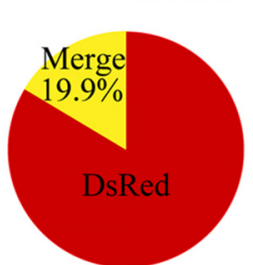

Figure 1. IC excitatory and inhibitory neurons as starter cells for rabies-based monosynaptic tracing. A, Characterization of Vglut2-Cre mice with Vglut2 ISH (top row) and VGAT-Cre mice with VGAT ISH (bottom row). Almost all EGFP-positive neurons expressed Vglut2 mRNA in Vglut2-Cre mice (top, arrowheads) and VGAT mRNA in VGAT-Cre mice (bottom, arrowheads). $\boldsymbol{B}$, Construction of the AAV helper virus and rabies virus (left) and time course of the experiment (right). C, Schematic diagram of extrinsic connections to the IC.(Figure legend continues.) 
correlation matrix (in which the rows and columns were equal to the input nuclei) was then used for the creation of a cluster tree using the built-in linkage function (a matrix in which the rows were cases and columns were distance between nodes). Finally, the built-in dendrogram function was used to display the tree (Altman and Krzywinski, 2017). To compare the difference between Vglut2-Cre and VGAT-Cre mice, we used a two-tailed unpaired $t$ test or Mann-Whitney $U$ test or one-way or two-way ANOVA with Prism software (GraphPad). All the $p$ values mentioned were post hoc tested and corrected with Holm-Sidak, Tukey, and Bonferroni methods. Error bars indicate the SEM.

\section{Results}

IC excitatory and inhibitory neurons as starter cells for rabies-based monosynaptic tracing

To target excitatory neurons in the IC, we used the Vglut2-Cre line, as IC excitatory neurons express Vglut2 but not Vglut1 (Ito et al., 2011). To target the GABAergic inhibitory population, we used the VGAT-Cre line, as glycinergic neurons, which also express VGAT, are absent in the IC (Tanaka and Ezure, 2004). To verify the specificity of the Cre lines in the IC, we injected Vglut2or VGAT-Cre mice with rAAV-EF1 $\alpha$-DIO-His-EGFP-2a-TVAWPRE-pA alone and then used ISH to examine the expression of Vglut2 or VGAT mRNA in infected neurons, respectively. Most of the AAV-infected neurons (Fig. 1A, green) in the Vglut2-Cre $(93 \pm 4 \%$; mean $\pm \mathrm{SD})$ and VGAT-Cre $(92 \pm 2 \%$; mean $\pm \mathrm{SD})$ lines were positive for the corresponding mRNA (Fig. 1A, red), demonstrating the high specificity of this method.

To achieve cell-type-specific monosynaptic tracing of extrinsic inputs, we used the modified monosynaptic rabies tracing technique, which has been widely used to characterize the presynaptic inputs of desired starter neurons with high accuracy and efficiency (Wickersham et al., 2007; Watabe-Uchida et al., 2012; Weissbourd et al., 2014; Menegas et al., 2015; Do et al., 2016). Briefly, the RV used here was modified to lack the ability to infect mammalian cells and its retrograde transsynaptic property by pseudotyping the virus with avian sarcoma leucosis virus envelop protein and replacing the transsynaptic required RG with DsRed (Fig. 1B). Cre-dependent AAV helper viruses encoding TVA, a receptor for avian sarcoma leucosis virus, and RG were used to label the starter neurons in the desired cell type, after which the RV was introduced to label their monosynaptic inputs (Fig. 1C). Starter neurons were identified by the coexpression of EGFP and DsRed, and input neurons were identified by the expression of DsRed (Fig. 1D, right).

To rule out nonspecific labeling, we performed control experiments. Injecting only rAAV-EF1 $\alpha$-DIO-RG-WPRE-pA and RV

$\leftarrow$

(Figure legend continued.) Cre-expressing-infected cells express EGFP-TVA (green dots) and RG. Neurons that contact these neurons are infected retrogradely and express DsRed alone (red dots, input neurons). Neurons expressing both EGFP and DsRed are the starter neurons (yellow dots). $\boldsymbol{D}$ Left, An image of the IC showing the input (red) and starter neurons (yellow) superimposed on the drawing of the IC subdivisions. The ICC (central IC) is the core, and the ICD (dorsal IC) and ICX (external IC) are grouped as the shell. The DsRed fluorescence intensity was enhanced to emphasize the labeled fibers primarily located in the ICD and ICX. Right, Starter neurons were identified by colocalization of EGFP (green) and DsRed (red) protein. $\boldsymbol{E}$, Injection of AAV-DIO-RG and RV without prior AAV-DIO-EGFP-TVA injection in the Vglut2-Cre mice resulted in no DsRed-labeled neurons. $F$, Injection of AAV-DIO-EGFP-TVA and RV without prior AAV-DIO-RG injection in the Vglut2-Cre mice resulted in no DsRed-labeled input neurons. $\mathbf{G}$, Injecting AAV-DI0-EGFP-TVA, AAV-DIO-RG, and RV to the wild-type mice and performed ISH for VGAT mRNA to identify the GABAergic neurons in the injection site. $\boldsymbol{H}$, Non-Cre, nonspecificlabeled RV neurons that expressed DsRed in the injection site. I, GABAergic neurons that identified by ISH. J, Six RV neurons (arrowheads) among the 27 RV neurons were GABAergic. $\boldsymbol{K}$, The $19.9 \%$ proportion of Cre-independent RV neurons was GABAergic ( 14 brain slices collected from 4 mice). Scale bars: $\boldsymbol{A}, \boldsymbol{H}, \boldsymbol{I}, \boldsymbol{J}, 20 \mu \mathrm{m} ; \boldsymbol{D}$, Left, $250 \mu \mathrm{m} ; \boldsymbol{D}$, Right, $50 \mu \mathrm{m} ; \boldsymbol{E}, \boldsymbol{F}, 200 \mu \mathrm{m}$. did not result in DsRed-labeled neurons, indicating the dependence of the RV infection on the AAV-induced expression of TVA (Fig. 1E). Injecting only rAAV-EF1 $\alpha$-DIO-His-EGFP-2aTVA-WPRE-pA and RV resulted in starter neurons that expressed both EGFP and DsRed without extrinsic input neurons, demonstrating that RG was necessary for the retrograde transport of the RV from the starter neurons (Fig. $1 F$ ). Injecting rAAV-EF1 $\alpha$-DIO-His-EGFP-2a-TVA-WPRE-pA, rAAV-EF1 $\alpha$ DIO-RG-WPRE-pA, and RV resulted in DsRed-labeled neurons in the injection site of wild-type mice (Fig. $1 G, H$ ). This is because very low-level expression of TVA in AAV-infected non-Cre neurons was sufficient for RV infection (Watabe-Uchida et al., 2012; Do et al., 2016). However, the extent of RV expression in non-Cre neurons was 10 times smaller than RV expression in Cre starter neurons ( $p<0.0001$; Mann-Whitney $U$ test; Vglut2: $1080 \pm$ $123, N=17$ vs wild-type: $81 \pm 8, N=6$; mean \pm SEM).

We performed further control experiments to show that each cell type was equally susceptible to the nonspecific labeling. We performed ISH for VGAT mRNA to identify the GABAergic nonCre, nonspecific labeling RV neurons of injection site (Fig. 1I). We found that the $19.9 \%$ proportion of Cre independent RV expressed neurons was GABAergic (Fig. $1 \mathrm{~J}, K$ ); this was quite consistent with the percentage of GABAergic neurons in the IC (Merchán et al., 2005; Beebe et al., 2016), suggesting that neither GABAergic neurons nor glutamatergic neurons were more susceptible to nonspecific labeling. Although the nonspecificlabeled RV neurons could be misidentified as local input neurons, the leaky expression of RG in non-Cre neurons was insufficient to cause transsynaptic infection of RV, as we did not observe any DsRed ${ }^{+}$neurons outside the injection site (data not shown). This was consistent with previous studies (Wall et al., 2013; Callaway and Luo, 2015; Do et al., 2016). Because of this, we primarily focused our analysis on the extrinsic inputs (except for Fig. $5 B$, which includes the intersubdivision inputs; and Fig. $9 B$, which includes intrinsic inputs; see below).

Because each IC subdivision receives a different combination of inputs from extrinsic nuclei, we divided cases into core, shell, and mixed injection cases based on the proportion of input neurons in each IC subdivision. We had 5 core and 7 shell cases in Vglut2-Cre mice and 4 core and 6 shell cases in VGAT-Cre mice (Fig. 2A,B). In the following results and discussion, the Vglut2Cre and VGAT-Cre cases are simply indicated by the discussion of either excitatory or inhibitory neurons, respectively.

To examine the variability of the viral retrograde tracing among different mice, we next counted the number of starter and input neurons (Fig. 2C,D) and performed a regression analysis for Vglut2-Cre, VGAT-Cre, and combined cases (Fig. 2E). The number of presynaptic input neurons had a linear relationship (Vglut2: $F_{(1,15)}=13.4$; VGAT: $F_{(1,15)}=5.2$; combined: $F_{(1,32)}=$ $6.2)$ to the number of starter neurons, suggesting that the input/ starter ratio was not related to the location of the injection and that this method was rather stable.

\section{Brainwide monosynaptic inputs to IC excitatory and inhibitory neurons}

To map the brainwide inputs to the IC, we grouped the extrinsic input neurons by anatomical locations. Here, we show an example of input neurons (red) to the IC and output fibers (green) from the IC of a Vglut2-Cre mouse (Fig. 3). Of the ascending nuclei, input neurons were found in the $\mathrm{CN}$, SOC, and nuclei of the LL (Fig. 3A-E). We confirmed that the input neurons in the contralateral (c) VCN (Fig. 3A) were VGAT-negative (Fig. 3B), consistent with previous results that $\mathrm{IC}$-projecting $\mathrm{CN}$ neurons 
A

Caudal<smiles>C1CC2CCC(C1)C2</smiles>

1
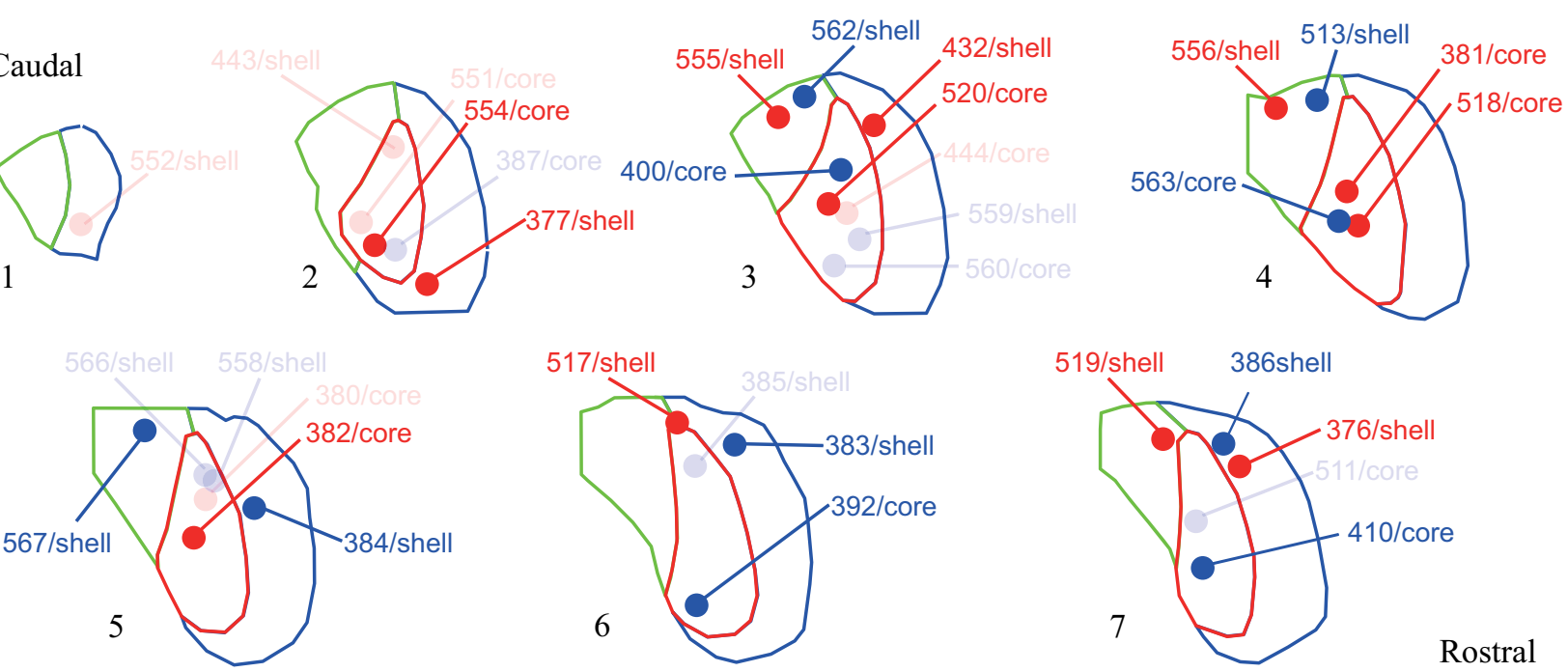

517/shell
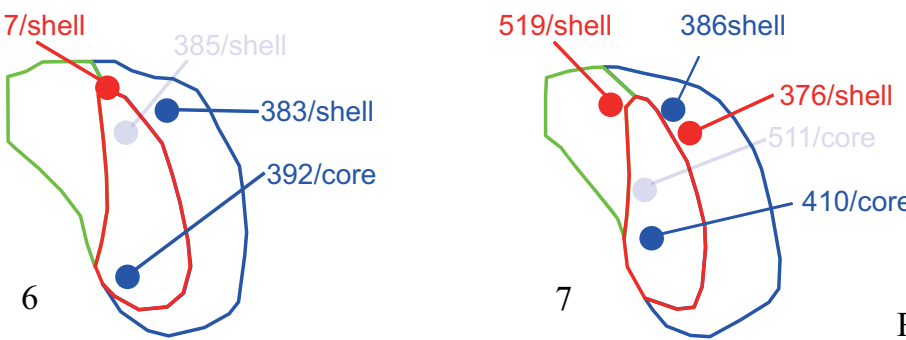

Rostral

B

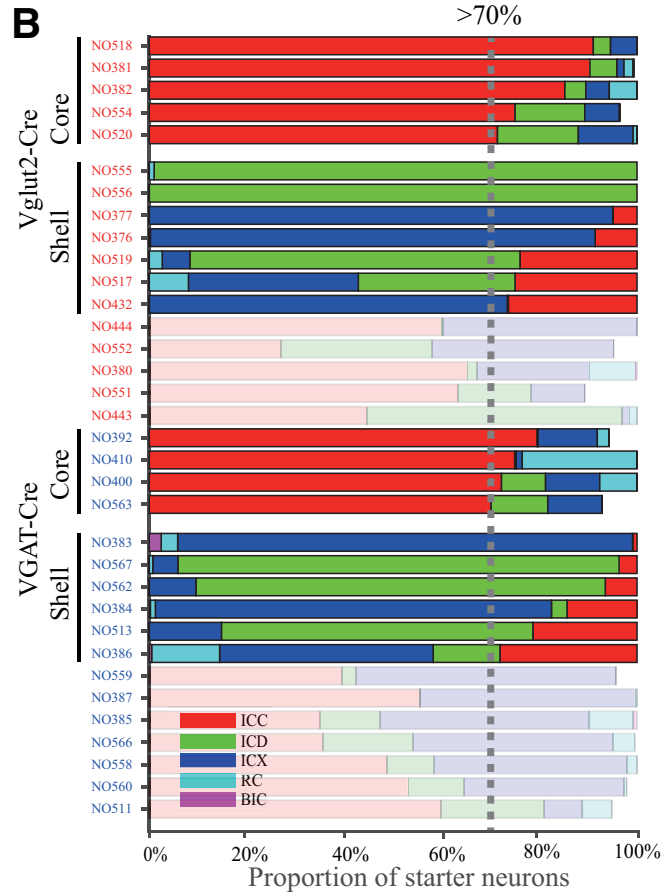

C

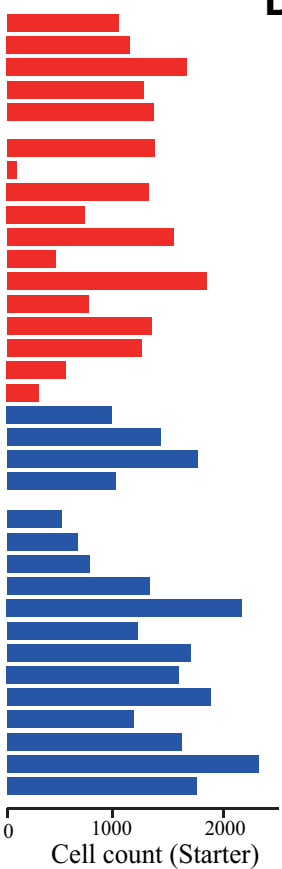

D
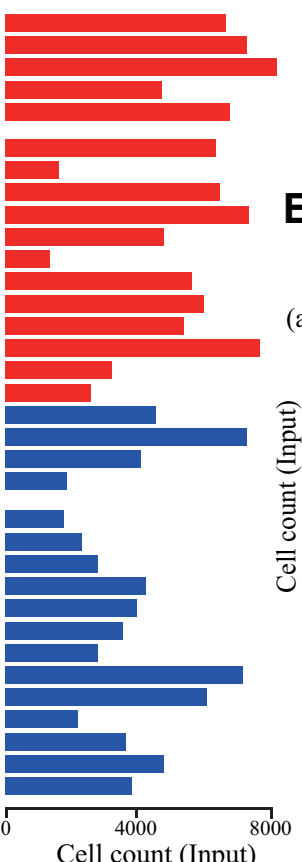

E $\mathrm{r}=0.69, \mathrm{p}=0.0023, \mathrm{slope}=2.61 \pm 0.71$ $\mathrm{r}=0.51, \mathrm{p}=0.0382$, slope $=1.47 \pm 0.65$ (all) $\mathrm{r}=0.40, \mathrm{p}=0.0181$, slope $=1.38 \pm 0.55$

Vglut2-Cre VGAT-Cre

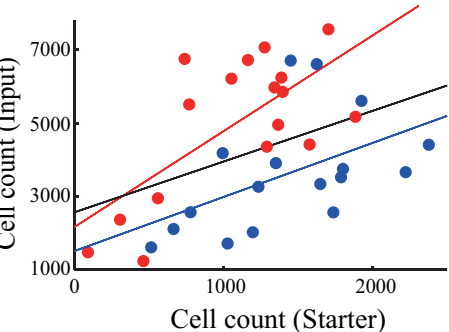

Figure 2. Brainwide monosynaptic inputs to IC excitatory and inhibitory neurons. $\boldsymbol{A}$, The distribution of the injection center of all cases examined in this study. Red dots indicate Vglut2-Cre cases. Blue dots indicate VGAT-Cre cases. Numbers indicate the animal ID (see also Fig. 2B). B, Core and shell cases of Vglut2-Cre and VGAT-Cre mice. Cases in which the proportion of starter cells in the ICC and IC shell met the 70\% threshold were classified as core and shell cases, respectively. The remaining cases with a lower threshold are shown with a faint color $(\boldsymbol{A}, \boldsymbol{B})$. $\boldsymbol{C}$, The number of starter neurons ranged from 89 to 2377 . $\boldsymbol{D}$, The number of input neurons ranged from 3482 to 13,208 . $\boldsymbol{E}$, A linear relationship was detected between the number of starter and input neurons.

are excitatory (Ito et al., 2015), and that the input neurons in the ipsilateral (i) VLL (Fig. 3D) were VGAT-positive (Fig. 3E), consistent with previous results that iVLL inputs to the IC are inhibitory (Saint Marie and Baker, 1990). Of the descending nuclei, thalamotectal inputs originated from the PP/PIL region, which was targeted by a small fraction of EGFP-positive IC ascending fibers (Fig. 3F, left). Cortical inputs originated mainly from layer 5 pyramidal neurons, fewer originating from L6 in the ipsilateral $\mathrm{AC}$ (Fig. 3F, middle and right). Fewer number input neurons were found in the ipsilateral temporal association area (Fig. $3 F$, right). Of the neuromodulatory nuclei, DsRed-positive input neurons as well as a few EGFP-positive IC projection fibers (Fig. $3 G$, left) were identified in the dorsal raphe, which is populated with serotonergic neurons. Likewise, in the SPF, the source of dopaminergic fibers in the IC, input neurons were also found with a few EGFP-positive fibers (Fig. $3 G$, middle).

The above examples (Fig. 3) show the inputs and outputs of IC excitatory glutamatergic neurons. To demonstrate the topographical and qualitative differences in inputs to different cell types of different IC subdivisions before quantitative analysis, here, 4 example cases were used to show the plots of starter cells and inputs cells of Vglut2-Cre and VGAT-Cre mice in the IC core (Fig. 4A,B) and shell (Fig. 4C,D), respectively. Overall, excitatory and inhibitory neurons in the same IC subdivisions received inputs from the same brain regions; and outside of the injection site, input neurons were densest in the contralateral IC and VLL. We excluded several nuclei from further analyses, including the hindbrain nuclei, ipsilateral $\mathrm{CN}$, contralateral VLL, and con- 
Ascending
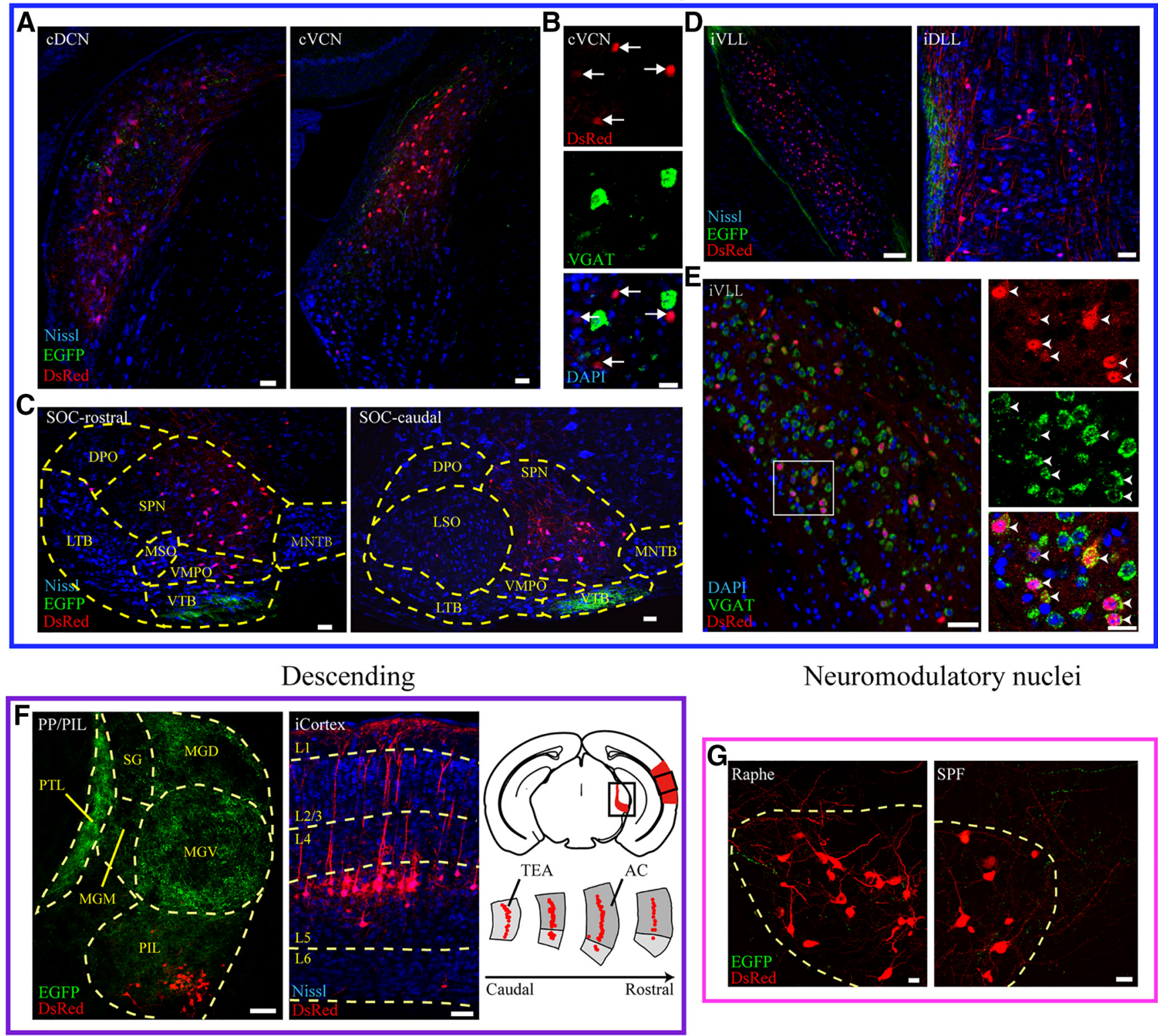

Neuromodulatory nuclei

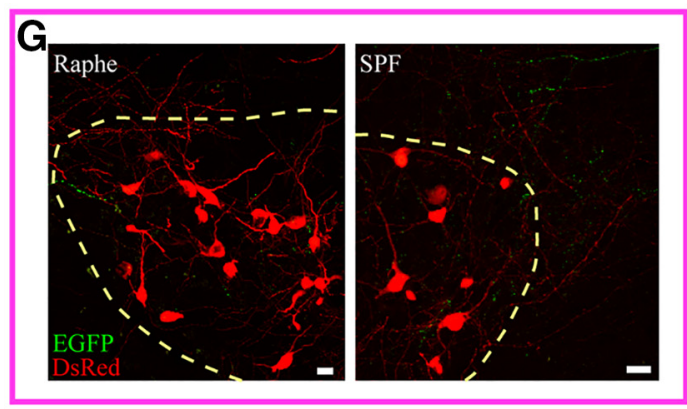

Figure 3. Characterization of the input neurons (red) and collicular fibers (green) in the ascending, descending, and neuromodulator nuclei of Vglut2-Cre mice. i, I psilateral inputs; $C$, contralateral inputs. $A$, Input neurons in the ascending nuclei, the CDCN and CVCN, with IC descending fibers. $B, C V C N$ input neurons (red, arrows) were negative for VGAT mRNA (green). $C$, Inputs from the ipsilateral SOC subnuclei. In the case shown here, input neurons were found in the LSO, MSO, and SPN, along with dense IC descending fibers in the VTB. DPO, Dorsal periolivary nucleus; VMPO, ventromedial periolivary nucleus; VTB, ventral nucleus of the trapezoid body; LTB, lateral nucleus of the trapezoid body; SPN, superior paraolivary nucleus; MNTB, medial nucleus of the trapezoid body. $\boldsymbol{D}$, Input neurons in ascending nuclei, the iVLL and iDLL, with IC descending fibers. $\boldsymbol{E}$, The iVLL input neurons were determined to be inhibitory due to the colocalization (arrowheads) of VGAT mRNA (green) with DsRed (red). $\boldsymbol{F}$, Left, Input neurons came from the PIL but were absent in the dorsal, medial, and ventral part of the medial geniculate nucleus (MGD, MGM, and MGV). SG, Suprageniculate thalamic nucleus; PTL, pretectothalamic lamina. Middle, Cortical input neurons from layer 5 (L5) and layer 6 (L6). Right top, Location of the auditory thalamus and cortex in the brain atlas. Right, bottom, Distribution of input neurons in the AC and temporal association area (TEA). G, Input neurons from neuromodulatory nuclei, the dorsal raphe (left) and SPF (right), with reciprocal IC projection fibers. Scale bars: $\boldsymbol{A}, \boldsymbol{C}, \boldsymbol{D}, \boldsymbol{E}$, Left, $50 \mu \mathrm{m} ; \boldsymbol{B}, 20 \mu \mathrm{m} ; \boldsymbol{E}$, Right, $20 \mu \mathrm{m} ; \boldsymbol{F}, 100 \mu \mathrm{m} ; \boldsymbol{G}, 10 \mu \mathrm{m}$.

tralateral AC because they only included a very small number of input neurons. Inputs from other SOC nuclei (PAG, cuneiform nucleus $\mathrm{CnF}$, and SC) were also excluded because their function in the IC is still largely unknown.

\section{Dominant ascending inputs to the IC shell subdivision}

IC shell excitatory neurons received more descending inputs from the AC than the IC core (Fig. $4 B, D$ ), which is consistent with the traditional view that the shell is the main recipient of the descending projection. However, the shell also received dense ascending inputs, especially from the VLL; as a result, the domi- nant inputs to the IC core/shell on excitatory/inhibitory neurons remained unclear. To resolve this issue, we analyzed the ratio of descending to ascending inputs. Although the shell glutamatergic neurons did not have a significantly higher ratio than the core glutamatergic or shell GABAergic neurons $(p=0.0600, p=$ 0.2248 , respectively) (between subdivision: $F_{(1,18)}=6.0, p=$ 0.0244 ; between cell type: $F_{(1,18)}=1.8, p=0.1984$; two-way ANOVA with Bonferroni correction), the ratio (0.3632 \pm 0.0392 ; mean \pm SEM) was still far $<1\left(t_{(6)}=6.1, p=0.0008\right)$ (Fig. $5 A$ ), indicating that the shell glutamatergic cells received more ascending than descending inputs. This difference in ascending and 
A
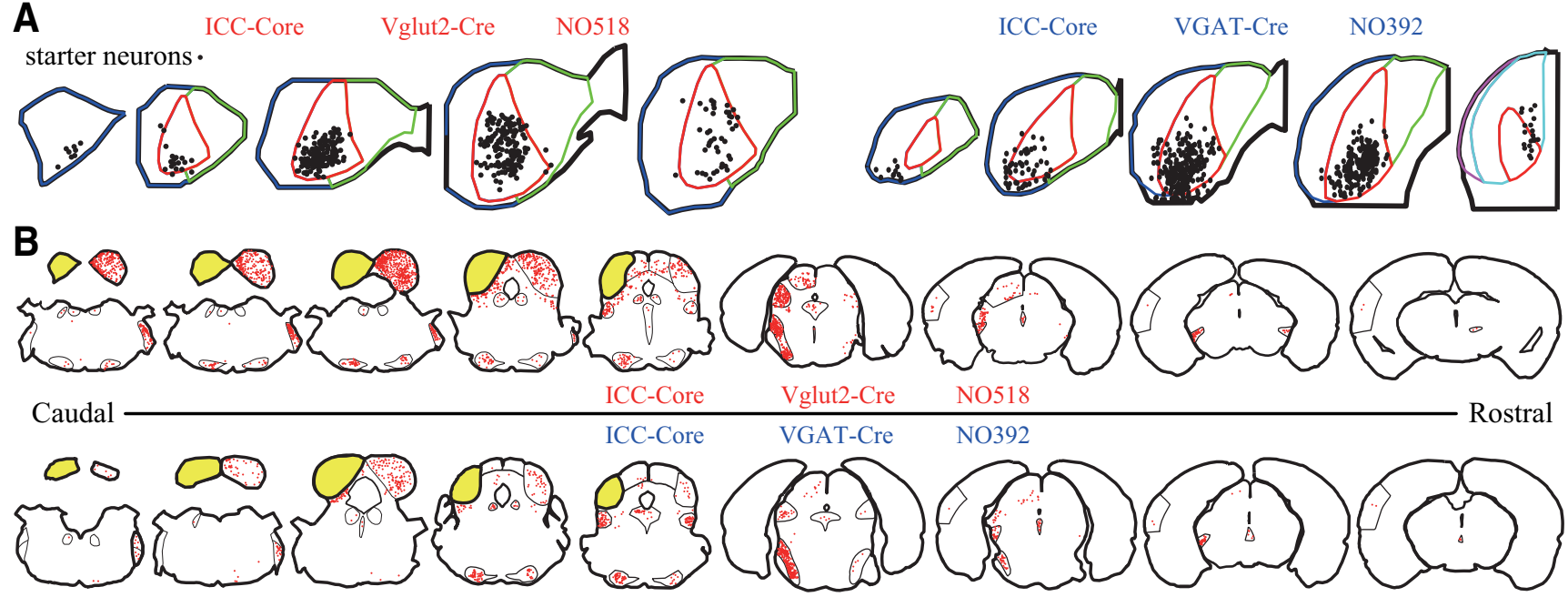

C
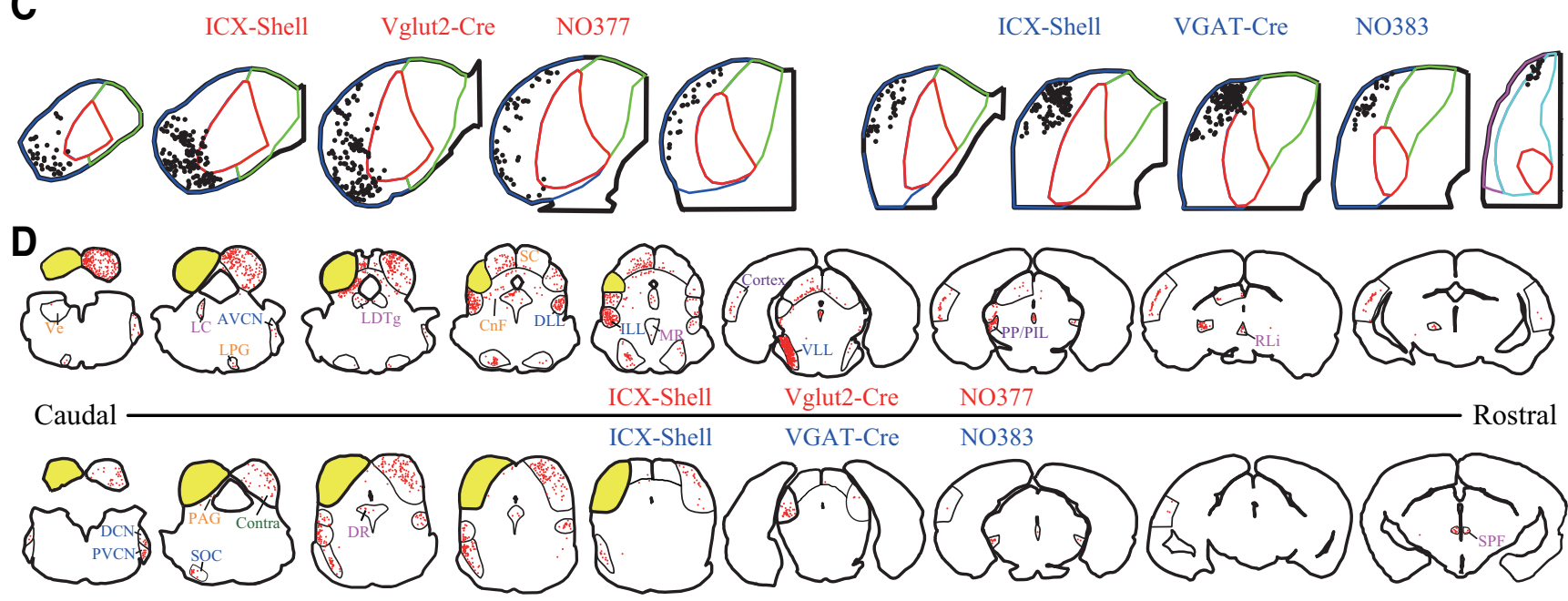

ICX-Shell

Vglut2-Cre

$\mathrm{NO} 377$

ICX-Shell VGAT-Cre NO383

Rostral
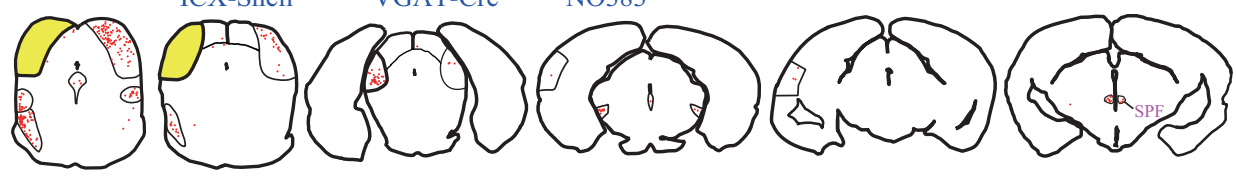

Figure 4. Topographical distribution of the starter and input neurons. Representative starter neurons $(\boldsymbol{A})$ and input neurons $(\boldsymbol{B})$ of corresponding core and shell cases $(\boldsymbol{C}, \boldsymbol{D})$ for $V g$ lut2-Cre and VGAT-Cre mice. Yellow represents the ipsilateral IC, which contains starter neurons. Red dots indicate input neurons.

descending inputs cannot be accounted for by the leakage of starter neurons into the core, which mainly received ascending inputs, as the two shell cases without any starter neurons in the core subdivision (Fig. 5A, larger dots) still predominantly received ascending inputs (i.e., the ratio was still $<1$ ).

In addition to ascending and descending inputs, neurons in the shell are also heavily innervated by intrinsic inputs from the ipsilateral IC core. Therefore, we normalized the ascending, descending, and core inputs by the corresponding number of starter neurons to reflect the average number of input neurons per starter cell. We found that shell glutamatergic neurons received more ascending inputs than descending $(p=0.0066)$ and core inputs $(p=0.0136)$, and the difference between the number of descending and core inputs was not significant $(p=0.9389)$ $\left(F_{(2,18)}=7.5, p=0.0042\right.$; one-way ANOVA with Tukey correction; Fig. 5B, left). Similarly, shell GABAergic neurons received more ascending inputs than descending $(p<0.0001)$ and core inputs $(p=0.0003)$, and the difference between the number of descending and core inputs was not significant $(p=0.2518)$ $\left(F_{(2,15)}=25.8, p<0.0001\right.$; one-way ANOVA with Tukey correction; Fig. $5 B$, right). In general, our results strongly suggested that ascending inputs far exceed descending and core inputs to neurons in the shell subdivision.
In the classical model, neurons within the IC shell subdivision receive more inputs from descending or core rather than ascending sources (Fig. 5C). In the new model introduced from the quantitative analysis described here, we propose that excitatory and inhibitory neurons within the IC shell subdivision receive more ascending inputs than descending or core inputs (Fig. 5D).

\section{IC core and shell excitatory neurons receive inputs from} different combinations of nuclei

Previous studies showed that small groups of neurons within a specific small region of the core or shell subdivisions mainly receive a particular combination of inputs from several nuclei (Loftus et al., 2010; Ayala et al., 2015). However, the postsynaptic cell types of such clustered inputs were unknown. To address this question, we performed correlation analyses of cells in the IC core and shell across Vglut2-Cre and VGAT-Cre mice and constructed dissimilarity cluster trees to reveal the input nuclei that were more related than others (Weissbourd et al., 2014; Menegas et al., 2015). This analysis relied on the fact that each injection likely covered a subset of neurons rather than targeting all IC neurons homogeneously. For example, in the IC core, a particularly strong negative correlation $\left(F_{(1,3)}=25.4\right)$ was seen between ascending cDCN and iLSO inputs to excitatory neurons (Fig. 6A, left), con- 
sistent with previous studies (Cant and Benson, 2006; Loftus et al., 2010). On the other hand, for inhibitory neurons, the same pair of nuclei were positively correlated $\left(F_{(1,2)}=18.6\right)$ (Fig. $6 \mathrm{~A}$, right).

We found that the input nuclei of the core glutamatergic neurons formed a cluster and displayed a large dissimilarity index with the clusters formed by other groups (Fig. $6 B$, top). In contrast, for core GABAergic neurons, most input nuclei were strongly correlated and displayed smaller dissimilarities between one another (Fig. 6B, bottom). Together, our results suggested that excitatory neurons receive inputs from different combinations of specific nuclei and inhibitory neurons receive inputs from the same combination of all nuclei in the core subdivision.

Although we previously found that shell excitatory neurons predominantly received ascending inputs and the largest descending inputs (Fig. $5 A, B$ ), whether those inputs converged onto one population or targeted two subpopulations was unknown. Here, we found that shell glutamatergic neurons that received inputs from the combination of ascending nuclei ("I") were distinct from those that received inputs from other nuclei ("II") (Fig. 6C, top), suggesting that those inputs were mostly segregated. On the other hand, inputs to shell GABAergic neurons displayed larger dissimilarities between each other and did not form clear clusters (Fig. 6C, bottom). We suggest that a bipartite domain, composed of the deep shell domain and nonlemniscal domain, is organized by the IC shell excitatory neurons (Fig. 6D) (see Discussion).

\section{Proportion of extrinsic inputs is different between excitatory and inhibitory neurons}

The ratio of inputs per starter neuron (i.e., the absolute number of inputs for specific cell types) revealed the degree of convergence of the extrinsic nuclei (Figs. 5, 6). On the other hand, the responses of a neuron to sound and other stimuli are more likely associated with the relative input strength of each nucleus, which may be represented by the proportion of inputs (Loftus et al., 2010). To this end, we normalized the inputs of each nucleus by the total number of extrinsic inputs and compared the ratio separately in the IC core and shell (Fig. 7A).

We first describe the results for the ascending group of inputs: compared with GABAergic neurons, glutamatergic neurons received a higher proportion of $\mathrm{cVCN}$ inputs in the core $(p<$ 0.0001 ) (between cell type: $F_{(1,63)}=0.3, p=0.5592$; between nuclei: $F_{(8,63)}=140.7, p<0.0001$; two-way ANOVA with Bonferroni correction) and tended to receive a lower proportion of cVCN inputs in the shell $(p=0.3475 ; 0.03 \pm 0.004$ vs $0.06 \pm$ 0.009 ; mean \pm SEM; between cell type: $F_{(1,99)}=0.6, p=0.4358$; between nuclei: $F_{(8,99)}=36.0, p<0.0001$; two-way ANOVA with Bonferroni correction) (Fig. 7A). We also found that GABAergic neurons received a higher proportion of iVLL inputs than glutamatergic neurons in the core $(p=0.0002)$ (Fig. $7 A)$. Next, we describe the analysis of the descending group of inputs: the glutamatergic neurons tended to receive a higher proportion of cor- tical inputs than GABAergic neurons in the shell subdivision $\left(t_{(11)}=2.0, p=0.0748 ; t\right.$ test; $0.07 \pm 0.006$ vs $0.03 \pm 0.003$; mean \pm SEM) (Fig. $7 A)$. Last, we describe the inputs from the neuromodulatory nuclei: in the IC core, GABAergic neurons received a higher proportion $\left(t_{(6)}=12.2, p<0.0001\right)$ of inputs from the raphe than glutamatergic neurons, whereas glutamatergic neurons received a higher proportion $\left(t_{(6)}=9.1, p=0.0001\right.$; all $t$ test) of inputs from the SPF than GABAergic neurons. In the IC shell, glutamatergic neurons received a higher proportion $\left(t_{(11)}=2.3, p=0.0435\right)$ of inputs from the locus ceruleus, the source of noradrenergic fibers, and LDTg/PPTg $\left(t_{(11)}=4.2\right.$, $p=0.0014$; all $t$ test), the source of cholinergic fibers, than GABAergic neurons (Fig. 7A).

When examining the outputs to the midbrain, we found that shell GABAergic neurons projected to the PAG (Fig. 7B, left), whereas glutamatergic neurons projected to both the PAG (Fig. $7 B$, right) and SC (Fig. $7 C$ ). In summary, the core GABAergic neurons received a lower proportion of $\mathrm{VCN}$ and higher proportion of VLL inputs than glutamatergic neurons, whereas shell GABAergic neurons tended to receive a higher proportion of VCN inputs and lower proportion of corticocollicular inputs than glutamatergic neurons (Fig. 7D). Importantly, the neuromodulatory nuclei differentially innervated glutamatergic and GABAergic neurons in the core and shell. Furthermore, all input nuclei, except for the cerebral cortex, received projections from the IC, suggesting a reciprocal relationship between the IC inputoutput systems.

Disinhibitory commissural connections and local circuits Previous studies have shown that glutamatergic and GABAergic neurons both project to the contralateral IC (Nakamoto et al., $2013 b$ ). Here, we found that projections of shell GABAergic neurons preferentially terminated in the contralateral dorsal shell 


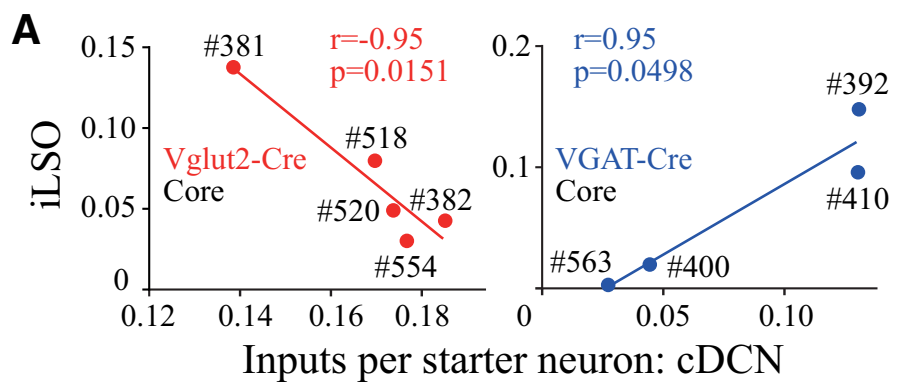

D

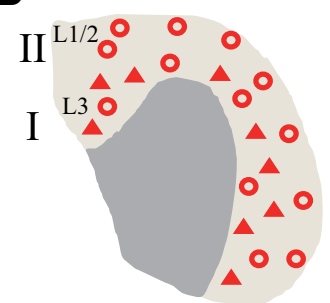

Ascending

Vglut2-Cre
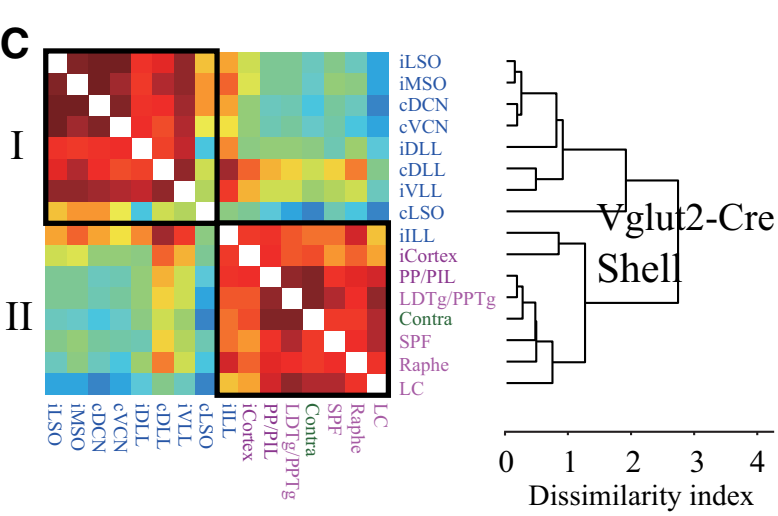

Dissimilarity index
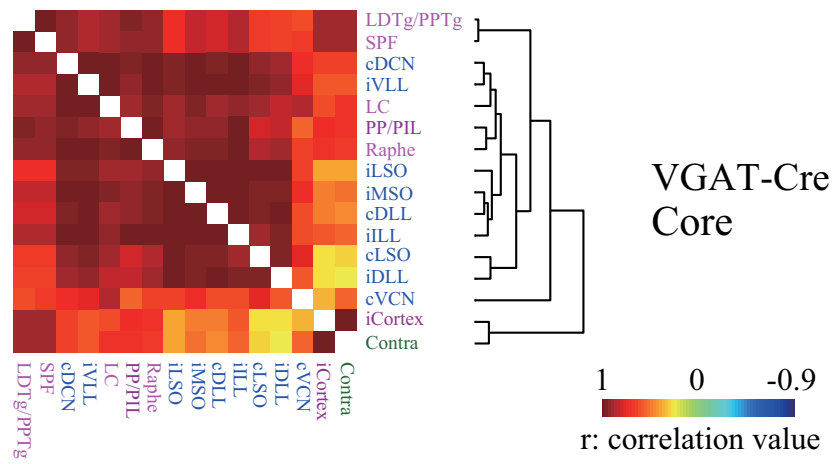
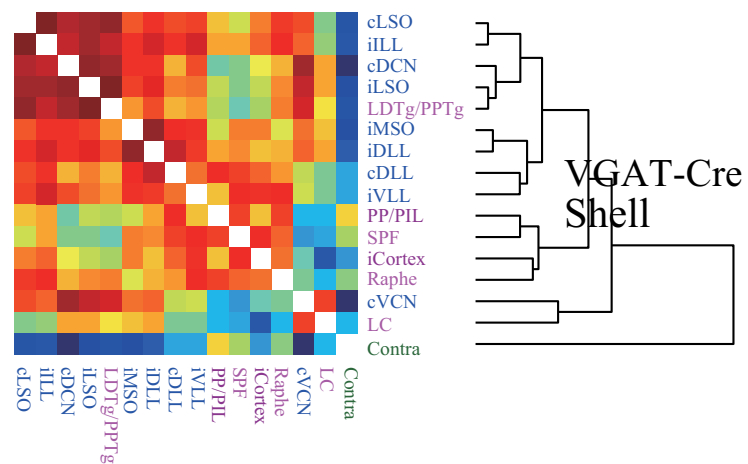

Figure 6. Glutamatergic neurons in the core and shell are subdivided into populations that receive inputs from different combinations of nuclei, whereas $G A B A e r g i c$ neurons in the core receive inputs from the same combination of nuclei. $A$, In the core subdivision, the iLSO and CDCN inputs are negatively correlated for glutamatergic neurons but positively correlated for GABAergic neurons. $r$, Pearson's correlation coefficient. Each dot indicates an individual mouse. $\boldsymbol{B}, \boldsymbol{C}$, Patterns of Pearson's correlation coefficient values and cluster trees showing the dissimilarities in input nuclei. In the core subdivision, three clusters of input nuclei that shared a high correlation were found for glutamatergic neurons, suggesting three populations that received inputs from different combinations of nuclei ( $\boldsymbol{B}$, top), whereas all input nuclei shared a high correlation for GABAergic neurons, suggesting a single population that received inputs from the same combination of nuclei ( $\boldsymbol{B}$, bottom). Likewise, in the shell subdivision, two populations of glutamatergic neurons were identified that received inputs from different combinations of nuclei ( $\boldsymbol{C}$, top, "I" and "II"), whereas the GABAergic population was not separated as they received inputs from mixed combinations of nuclei (C, bottom). D, Suggested bipartite domain of glutamatergic neurons: The deep shell domain ("I" and triangles in L3) mainly received ascending inputs. In contrast, the nonlemniscal domain in the other part of the shell ("II" and open circles in L3 and L1/2) mainly received descending inputs.

subdivision (Fig. 8A), and the connection between both sides of the IC was homotopic for GABAergic neurons (Fig. 8B). Although one study revealed that the GABAergic neurons receive contralateral inhibitory inputs (Lee et al., 2015), the cell type of the IC commissural input neurons is less understood overall. Therefore, we performed ISH for VGAT mRNA to identify GABAergic commissural projection neurons and calculated the proportion of VGAT-positive input neurons to total commissural input neurons in the contralateral core and shell subdivision, respectively (Fig. 8C). The proportion of VGAT-expressing neurons that projected onto contralateral glutamatergic neurons was similar between the shell and core $\left(t_{(12)}=0.7, p=0.4759, t\right.$ test; $33.76 \pm 10.81 \%$ vs $37.65 \pm 6.05 \%$; mean $\pm \mathrm{SD}$ ) (Fig. $8 D$, left). However, the proportion of VGAT-expressing neurons that projected onto contralateral GABAergic neurons was significantly higher in the shell than in the core $\left(t_{(20)}=9.9, p<0.0001\right.$, $t$ test; $65.39 \pm 10.23 \%$ vs $22.09 \pm 4.26 \%$; mean \pm SD) (Fig. $8 D$, right). In general, our anterograde and retrograde results indicate that the commissural projection onto GABAergic neurons from the shell was mainly inhibitory and that from the core was mainly excitatory, suggesting that contralateral shell GABAergic neurons may inhibit ipsilateral GABAergic neurons and then disinhibit their efferent targets (Fig. 8E).

In addition to extrinsic inputs, excitatory and inhibitory neurons also received considerable local inputs (Fig. 9A). Here, we compared the ratio of intrinsic inputs with extrinsic inputs among core and shell, glutamatergic, and GABAergic neurons (Fig. 9B). We found considerable local and intersubdivision connections in shell neurons $\left(t_{(30)}=3.0, p=0.0060\right)$ and GABAergic neurons $\left(t_{(30)}=2.5, p=0.0163\right.$; all $t$ test $)$. Based on previous studies (Lesicko et al., 2016) and our results from the ascending (Fig. 7A), contralateral (Fig. 8), and local inputs (Fig. 9) to GABAergic neurons, we suggest a model that could partially explain the multisensory function of the ICX (Fig. 9C; see Discussion). 
A

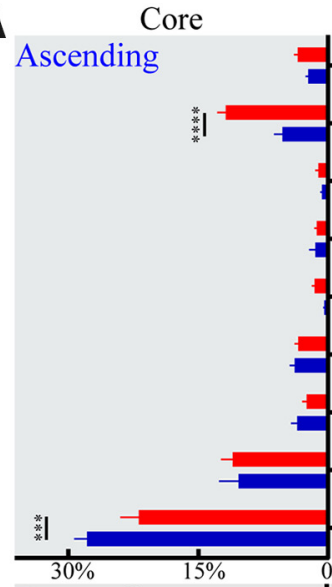

Descending

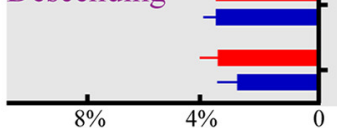

Neuromodulatory nuclei

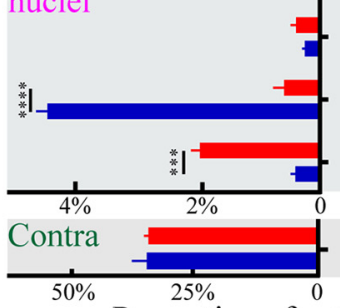

Shell

IVglut2-Cre vGAT-Cre
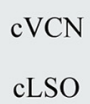

iLSO

iMSO

cDLL

iDLL

ilLL

iVLL
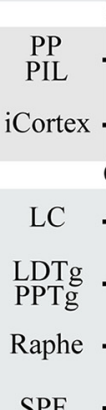

Contra
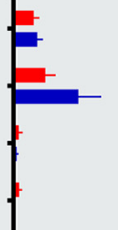

단
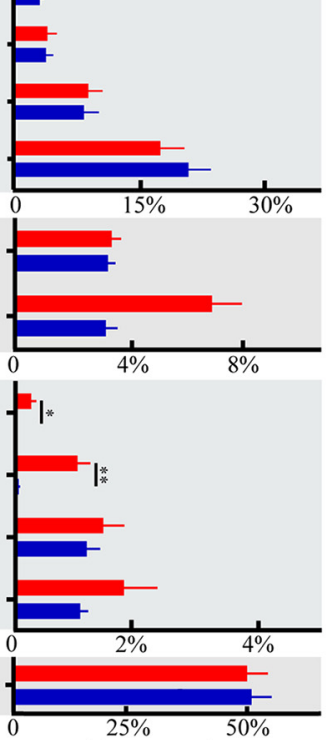

inputs (4 groups)
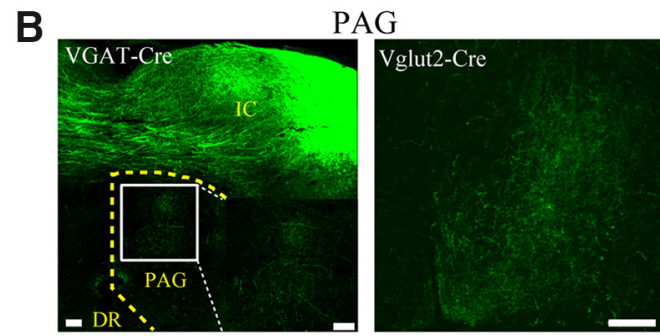

C

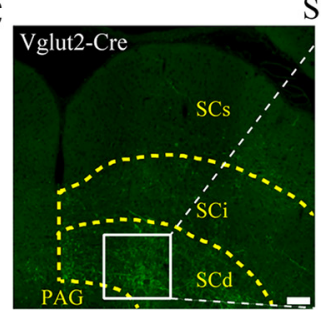

$\mathrm{SC}$

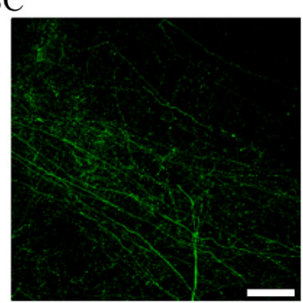

D

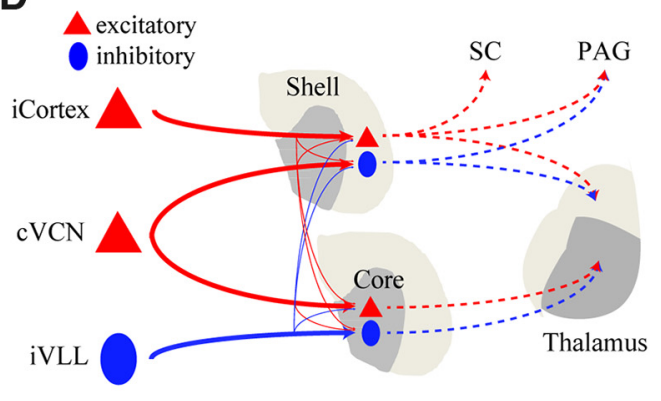

Figure 7. Extrinsic inputs and outputs differ between glutamatergic and GABAergic neurons. $A$, Summary of the proportion of extrinsic inputs from the nuclei categorized as ascending, descending, neuromodulatory nuclei, and contralateral IC. Bars represent the average percentage inputs of all input neurons per region (mean \pm SEM). The statistical significance was calculated from a two-way ANOVA with Bonferroni correction for the ascending group of nuclei and a multiple two-tailed unpaired $t$ test followed by Holm-Sidak correction for the other groups. $B, C$, Projections to the PAG and SC. B, Left, Projections to the PAG in VGAT-Cre mice. Inset, DR, Dorsal raphe. Magnified images show the axonal fibers within the PAG. Right, Projections to the PAG in Vglut2-Cre mice. C, Left, Projections to the SCd in Vglut2-Cre mice. Superficial, intermediate, and deep layer of the SC (SCS, SCi, and SCd). Right, Magnified images show the axonal fibers within the SCd. Scale bar, $100 \mu \mathrm{m}$. D, Schematic diagram drawn from the current results and previous studies. Red and blue represent soma and axon of excitatory and inhibitory neurons, respectively. Core GABAergic neurons received a higher proportion of inhibitory VLL inputs than excitatory neurons (wide vs slim solid line) and projected to the core thalamus (dotted line). Shell GABAergic neurons tended to receive a higher proportion of excitatory VCN inputs than glutamatergic neurons and projected to the shell thalamus and PAG. Core glutamatergic neurons received a higher proportion of VCN inputs than GABAergic neurons and projected to the core thalamus, whereas shell glutamatergic neurons tended to receive a higher proportion of cortical inputs than inhibitory neurons, and projected to the shell thalamus, PAG, and SC. ${ }^{*} p<0.05 ;{ }^{* *} p<0.01 ;{ }^{* * *} p<0.001 ;{ }^{* * *} p<0.0001$.

\section{Discussion}

In this study, we revisited the neuronal organization in the IC using monosynaptic and cell-type-dependent methods. We revealed that IC shell neurons predominantly received ascending inputs. Two subpopulations of IC shell excitatory neurons received inputs from different combinations of nuclei, implicating a bipartite domain in the IC shell. In the IC core, we found that the excitatory neurons received different combinations of inputs, whereas the inhibitory neurons received the same combination. In addition, we uncovered two long-range disinhibitory connections in the IC core and shell.

\section{Dominant ascending pathway in the IC shell}

In previous studies (Tokunaga et al., 1984; Coleman and Clerici, 1987), a retrograde tracer HRP was injected into the superficial layer of the IC shell subdivision in the rat and showed that the major source of inputs was from the somatosensory nuclei, IC core and AC. However, our study revealed that shell neurons predominantly received inputs from ascending auditory nuclei. Two differences may account for this discrepancy.
One difference may arise from different injection areas within the shell. Considerable ascending inputs were observed when the HRP injection covered both the ICC and ICX (Aitkin et al., 1981). However, a portion of the injection area assigned to the ICC in the above study was actually the ventrolateral nucleus in the cat, which corresponds to layer 3 of the ICX in the rat (Loftus et al., 2008). Furthermore, differences between the traditional tracer and RV methods (see Introduction) may also contribute to the discrepancy. Specifically, unlike the small- and medium-sized neurons of the superficial layer, the deep layer of the shell contains large multipolar neurons whose dendrites not only extend a long distance within the shell but also project into the adjacent core subdivision and are densely covered by spines (Malmierca et al., 2011). When the diffusion of the conventional retrograde tracer is limited locally, inputs to dendrites that are outside of the injection sites will be missed.

In addition, many anterograde tracing studies have clearly shown that the deep layer of the shell is innervated by numerous ascending fibers from auditory brainstem nuclei: that is, the $\mathrm{CN}$ (Oliver et al., 1999; Malmierca et al., 2005; Loftus et al., 2008), 

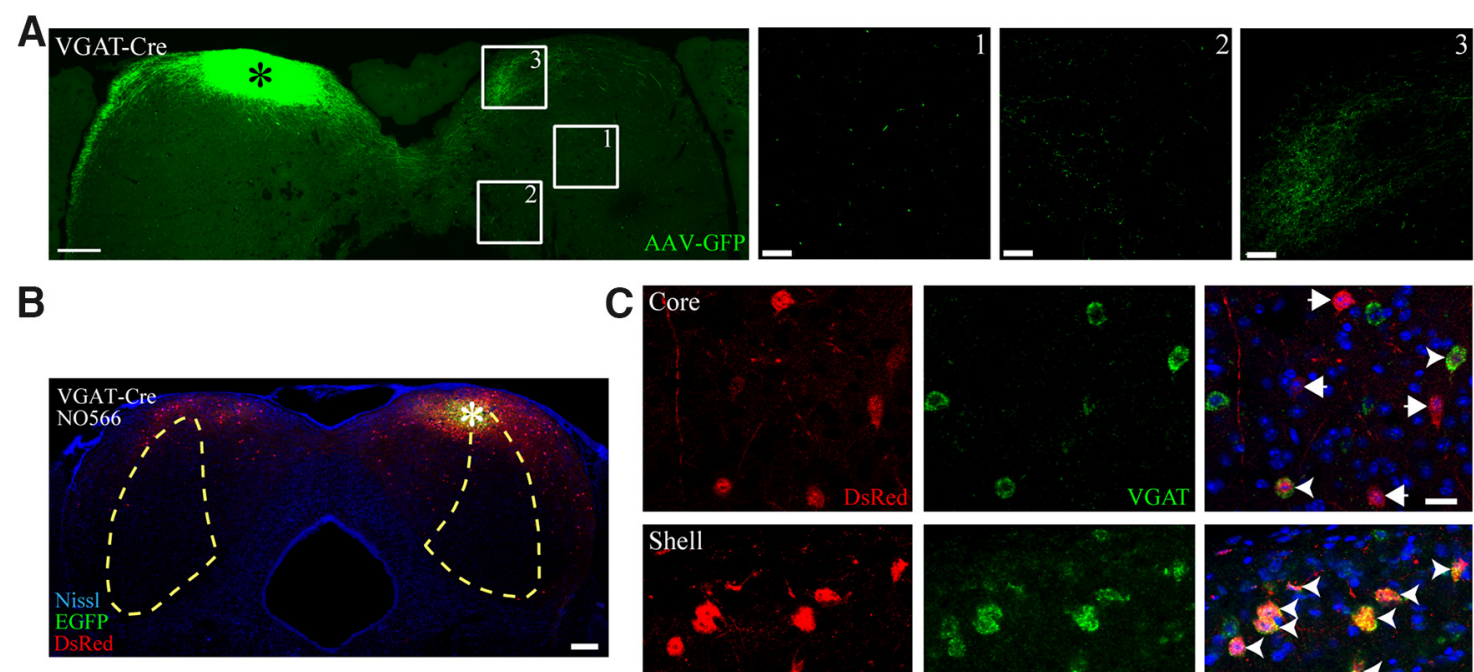

\section{Core}
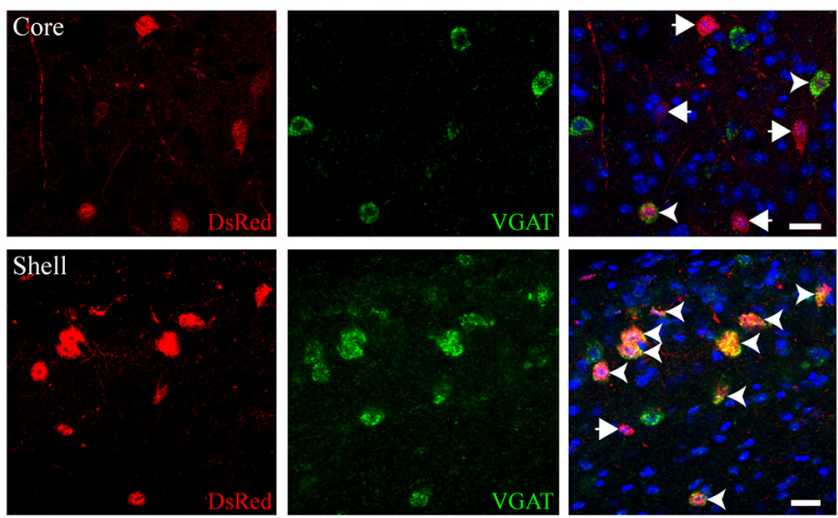

D
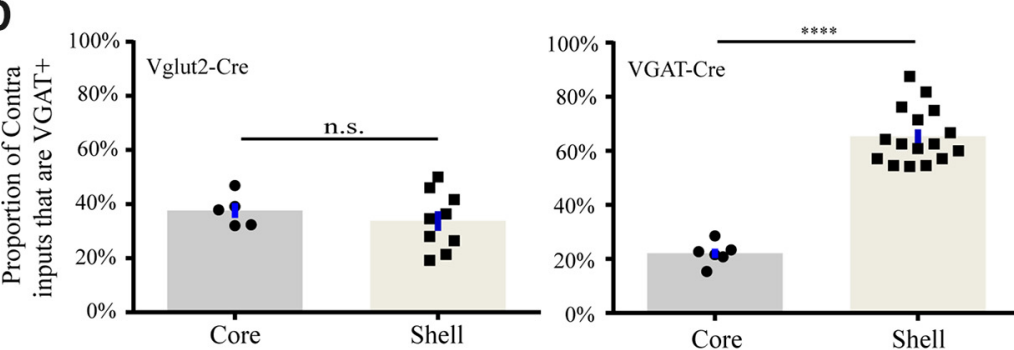

E

excitatory

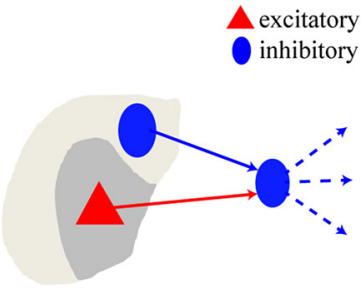

Commissural connections

Figure 8. Disinhibitory commissural connections. $A$, Left, Commissural projection pattern of shell GABAergic neurons. Right, Magnified images show the axonal fibers in the core ( 1 and 2 ) and shell (3). Denser fibers were found in the contralateral shell. B, Symmetrical contralateral input pattern in VGAT-Cre mice. C, ISH staining for VGAT mRNA (green) in contralateral input neurons (red) in a VGAT-Cre mouse. Top, A few VGAT-expressing input neurons (yellow) were found in the contralateral core. Bottom, Many of the input neurons expressed VGAT mRNA in the contralateral shell. Arrowheads indicate VGAT-positive. Arrows indicate VGAT-negative. Scale bars: $\boldsymbol{A}$, left, $250 \mu \mathrm{m} ; \boldsymbol{A}$, right, $\boldsymbol{C}, 20 \mu \mathrm{m} ; \boldsymbol{B}, 200 \mu \mathrm{m}$. * Location of the injection sites. $\boldsymbol{D}, \mathrm{GABAergic}$ neurons received a higher proportion of VGAT-positive input from the contralateral IC shell than glutamatergic neurons. ${ }^{* * *} p<0.0001$ (two-tailed unpaired $t$ test followed by Holm-Sidak correction). $E$, Inhibitory dominant projection in the shell (blue color solid line) and excitatory dominant projection in the core (red color solid line). Contralateral inhibition of ipsilateral GABAergic neurons could disinhibit their targets through efferent projections (blue color dotted line).

SOC (Willard and Martin, 1983; Loftus et al., 2004; Saldaña et al., 2009), and nuclei of the LL (Whitley and Henkel, 1984; Shneiderman et al., 1988; Gabriele et al., 2000). Accordingly, the difference is likely attributable to the fact that neurons in the deep layer of the shell or their long-range dendritic inputs were severely undersampled, if not entirely missed, in some previous studies.

\section{Implications for a bipartite domain in the IC shell}

Neurons within the IC shell displayed stronger SSA than those in the core (Shen et al., 2015), and Ayala et al. (2015) reported that IC shell SSA sites received descending and core inputs but almost completely lacked lemniscal inputs. In contrast, Syka et al. (2000) reported that shell neurons and core neurons exhibited similarly short response latencies. These observations are suggestive of two different domains in the shell. Consistent with this, our results suggested that a group of excitatory neurons in the shell primarily received lemniscal inputs (Fig. 6C, top "I") and were likely located within the deep layer of the shell (discussion above; Fig. $6 D$, "I" and triangle). In contrast, another group of IC shell neurons mainly received nonlemniscal inputs (Fig. 6C, top "II") and were likely located throughout all layers of the shell (Fig. 6D, "II" and open circle). Thus, we propose that a bipartite domain may exist in the IC shell that is composed of the deep shell domain and nonlemniscal domain (Fig. 6D).

\section{Excitatory neurons receive inputs from different} combinations of nuclei, whereas inhibitory neurons receive inputs from the same combination of nuclei in the IC core The synaptic domain or functional module hypothesis predicts that neurons with similar response properties will receive similar inputs (Oliver, 2005). In the well-studied core subdivision, for example, units with monaural and binaural responses mainly receive ascending inputs from corresponding nuclei (Loftus et al., 2010). However, AC descending projections to the core, which are topographically organized (Saldaña et al., 1996; Bajo et al., 2007), had been poorly examined in previous studies. Although ascending and descending projections both innervate the core, we found that neurons that received descending inputs were distinct from other neurons, especially those that received ascending inputs (Fig. 6B, top), consistent with the previous hypothesis that descending inputs collectively converge onto regions other than those innervated by ascending inputs (Winer et al., 1998). On the other hand, GABAergic neurons received inputs from the same combination of all nuclei (Fig. $6 \mathrm{~B}$, bottom). In the core, the majority of large cells that also exhibit large dendritic trees (Oliver et al., 1991) are GABAergic neurons (Beebe et al., 2016). In addition, we found that GABAergic neurons had more intrinsic connectivity than glutamatergic neurons (Fig. 9B). Therefore, single 
core inhibitory neuron may integrate all extrinsic and intrinsic inputs with longrange dendrites.

Two long-range disinhibition circuits Inhibitory neurons in the IC and VLL are well known for their ascending projections. The VLL provides the largest source of inhibitory ascending projections to the IC (Fig. 7A), and its neurons display either an onset or sustained response to sound stimuli, with those with an onset response showing a higher selectivity for temporally modulated sound (Zhang and Kelly, 2006). When projection fibers from the IC to medial geniculate body (MGB) are electrically stimulated, almost all MGB neurons are initially inhibited and then excited, indicating that IPSPs arrive before EPSPs (Peruzzi et al., 1997). Here, we found that core inhibitory neurons received a higher proportion of VLL inputs than excitatory neurons, suggesting that this inhibitory feedforward tectothalamic circuit may be effectively modulated by the VLL.

The IC shell is characterized by its multimodal connections. GABAergic modules in the ICX receive somatosensory inputs, whereas the extramodular regions receive auditory inputs (Lesicko et al., 2016). Furthermore, the ICX mainly receives ipsilateral somatosensory inputs (Coleman and Clerici, 1987; Zhou and Shore, 2006) but responds to bilateral stimuli (Robards, 1979), suggesting the convergence of bilateral inputs within the shell. Because somatosensory inputs can reduce neuronal responses to auditory stimulation (Jain and Shore, 2006), the auditory responses are likely inhibited by bilateral somatosensory inputs via GABAergic modules. Our results suggest some possible anatomical substrates for multisensory integration. First, shell inhibitory neurons tended to receive a higher proportion of ascending VCN inputs than excitatory neurons (Fig. $7 A$ ). Second, shell inhibitory neurons appeared to primarily innervate contralateral shell inhibitory neurons (Fig. $8 A, C, D$ ). Third, inhibitory neurons received more intrinsic inputs than excitatory neurons (Fig. 9B). Together, we hypothesize that the subsets of GABAergic neurons within the extramodular region are candidate substrates for multisensory integration and integrate excitatory auditory inputs from the VCN and inhibitory somatosensory inputs from bilateral GABAergic modules (Fig. 9C).

\section{Functional implications}

From the major findings of this study, we suggest the following three functional implications:

First, unlike the nonlemniscal domain that primarily receives descending inputs, the deep shell domain is predominated by ascending inputs (Fig. 6C, top). Unlike the lemniscal domain that primarily projects to MGB, this domain heavily innervates PAG and SC. From a neuroethological point of view (Casseday and Covey, 1996), the evidence suggests that deep shell domain relays ascending inputs and projects to the PAG and SC, to drive acoustic-motor behavior, as follows: escaping from a predator
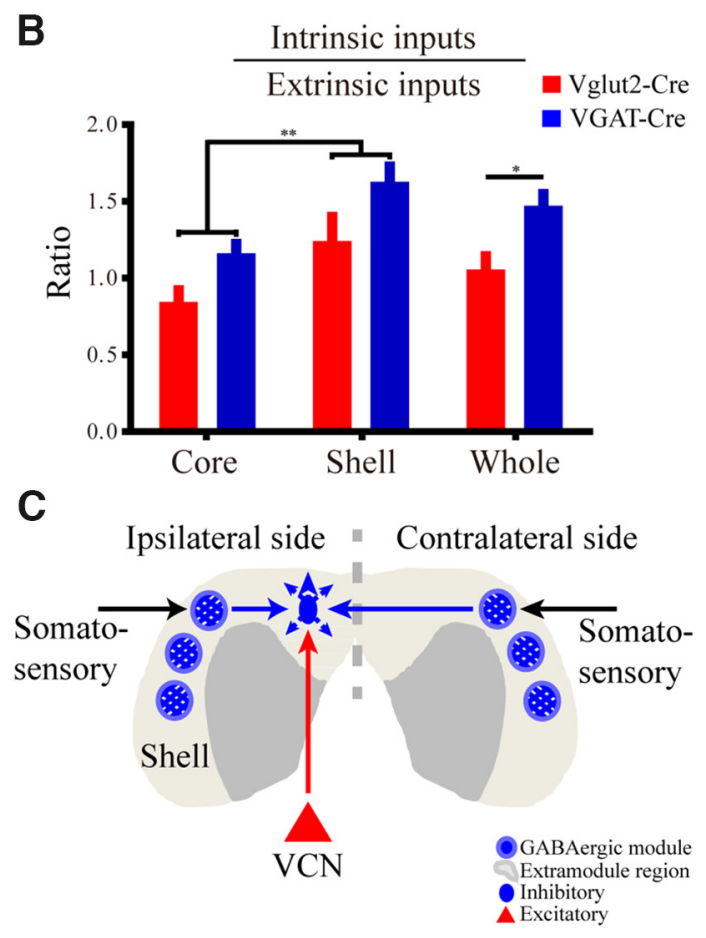

Figure 9. Local circuits. $\boldsymbol{A}$, V-shape intrinsic connection patterns of Vglut2-and VGAT-Cre mice with the approximate locations extramodular region, inhibitory neurons receive a higher proportion of ascending VCN inputs (red solid line), and shell inhibitory neurons mainly innervate contralateral shell inhibitory neurons (blue solid line that crossed midline). Those inhibitory neurons also receive more intrinsic inputs (blue solid line) and may project to neighbor neurons (blue dotted line).

(Xiong et al., 2015), the localization of prey (Knudsen and Konishi, 1978; King et al., 1998), and conspecific communication (Jürgens, 2002; Wilczynski and Ryan, 2010). Similarly, SC not only responds to visual stimuli but also drives visually innate behavior (Shang et al., 2015; Wei et al., 2015). Therefore, the IC and SC may share common circuitry that links sensory inputs with motor outputs directly.

Second, a recent study showed that responses of IC excitatory and inhibitory neurons to pure tones are similar (Ono et al., 2017). As core inhibitory neurons receive inputs from the same combination of nuclei and each input nucleus encodes different aspects of sound information, convergence of extrinsic inputs may produce de novo responses to the complex sound (e.g., dynamic moving ripple and communication calls) (Chen et al., 2012; Akimov et al., 2017) in the core inhibitory neurons. This contrasts with the AC, where inhibitory neurons exhibit more separable spectral and temporal receptive fields to the dynamic moving ripple stimuli (Atencio and Schreiner, 2008).

Last, disinhibition triggered by onset-type VLL neurons may release the fast IPSPs in the thalamus at the onset of a sound stimulus and enhance the precision of auditory temporal pattern processing in higher centers of the auditory system. In contrast, the sustained-type VLL neurons may release the thalamus from GABAergic inhibition and further relay information about the stimulus duration (Covey and Casseday, 1999). Therefore, inhibitory projections from the VLL to IC and then to the MGB highlight the role of long-range disinhibition for auditory temporal processing. Interestingly, long-range disinhibition circuits had 
recently also been observed in other brain areas (Caputi et al., 2013).

We hope this systematic and cell-type-defined study on IC neuroanatomy will lay the foundation for physiological and behavioral experiments that probe further functional differences between excitatory and inhibitory neurons.

\section{References}

Aitkin LM, Kenyon CE, Philpott P (1981) The representation of the auditory and somatosensory systems in the external nucleus of the cat inferior colliculus. J Comp Neurol 196:25-40. CrossRef Medline

Akimov AG, Egorova MA, Ehret G (2017) Spectral summation and facilitation in on- and off-responses for optimized representation of communication calls in mouse inferior colliculus. Eur J Neurosci 45:440-459. CrossRef Medline

Altman N, Krzywinski M (2017) Clustering. Nat Methods 14:545-546. CrossRef

Atencio CA, Schreiner CE (2008) Spectrotemporal processing differences between auditory cortical fast-spiking and regular-spiking neurons. J Neurosci 28:3897-3910. CrossRef Medline

Ayala YA, Udeh A, Dutta K, Bishop D, Malmierca MS, Oliver DL (2015) Differences in the strength of cortical and brainstem inputs to SSA and non-SSA neurons in the inferior colliculus. Sci Rep 5:10383. CrossRef Medline

Bajo VM, Nodal FR, Bizley JK, Moore DR, King AJ (2007) The ferret auditory cortex: descending projections to the inferior colliculus. Cereb Cortex 17:475-491. CrossRef Medline

Beebe NL, Young JW, Mellott JG, Schofield BR (2016) Extracellular molecular markers and soma size of inhibitory neurons: evidence for four subtypes of GABAergic cells in the inferior colliculus. J Neurosci 36:39883999. CrossRef Medline

Callaway EM, Luo L (2015) Monosynaptic circuit tracing with glycoprotein-deleted rabies viruses. J Neurosci 35:8979-8985. CrossRef Medline

Cant NB, Benson CG (2006) Organization of the inferior colliculus of the gerbil (Meriones unguiculatus): differences in distribution of projections from the cochlear nuclei and the superior olivary complex. J Comp Neurol 495:511-528. CrossRef Medline

Caputi A, Melzer S, Michael M, Monyer H (2013) The long and short of GABAergic neurons. Curr Opin Neurobiol 23:179-186. CrossRef Medline

Casseday JH, Covey E (1996) A neuroethological theory of the operation of the inferior colliculus. Brain Behav Evol 47:311-336. CrossRef Medline

Chen C, Rodriguez FC, Read HL, Escabí MA (2012) Spectrotemporal sound preferences of neighboring inferior colliculus neurons: implications for local circuitry and processing. Front Neural Circuits 6:62. CrossRef Medline

Coleman JR, Clerici WJ (1987) Sources of projections to subdivisions of the inferior colliculus in the rat. J Comp Neurol 262:215-226. CrossRef Medline

Covey E, Casseday JH (1999) Timing in the auditory system of the bat. Annu Rev Physiol 61:457-476. CrossRef Medline

Do JP, Xu M, Lee SH, Chang WC, Zhang S, Chung S, Yung TJ, Fan JL, Miyamichi K, Luo L, Dan Y (2016) Cell type-specific long-range connections of basal forebrain circuit. eLife 5:e13214. CrossRef Medline

Gabriele ML, Brunso-Bechtold JK, Henkel CK (2000) Development of afferent patterns in the inferior colliculus of the rat: projection from the dorsal nucleus of the lateral lemniscus. J Comp Neurol 416:368-382. CrossRef Medline

Geis HR, Borst JG (2013) Large GABAergic neurons form a distinct subclass within the mouse dorsal cortex of the inferior colliculus with respect to intrinsic properties, synaptic inputs, sound responses, and projections. J Comp Neurol 521:189-202. CrossRef Medline

González-Hernández T, Mantolán-Sarmiento B, González-González B, Pérez-González H (1996) Sources of GABAergic input to the inferior colliculus of the rat. J Comp Neurol 372:309-326. CrossRef Medline

Ito T, Oliver DL (2014) Local and commissural IC neurons make axosomatic inputs on large GABAergic tectothalamic neurons. J Comp Neurol 522:3539-3554. CrossRef Medline

Ito T, Bishop DC, Oliver DL (2009) Two classes of GABAergic neurons in the inferior colliculus. J Neurosci 29:13860-13869. CrossRef Medline

Ito T, Bishop DC, Oliver DL (2011) Expression of glutamate and inhibitory amino acid vesicular transporters in the rodent auditory brainstem. J Comp Neurol 519:316-340. CrossRef Medline

Ito T, Hioki H, Sohn J, Okamoto S, Kaneko T, Iino S, Oliver DL (2015) Convergence of lemniscal and local excitatory inputs on large GABAergic tectothalamic neurons. J Comp Neurol 523:2277-2296. CrossRef Medline

Jain R, Shore S (2006) External inferior colliculus integrates trigeminal and acoustic information: unit responses to trigeminal nucleus and acoustic stimulation in the guinea pig. Neurosci Lett 395:71-75. CrossRef Medline

Jürgens U (2002) Neural pathways underlying vocal control. Neurosci Biobehav Rev 26:235-258. CrossRef Medline

King AJ, Jiang ZD, Moore DR (1998) Auditory brainstem projections to the ferret superior colliculus: anatomical contribution to the neural coding of sound azimuth. J Comp Neurol 390:342-365. CrossRef Medline

Knudsen EI, Konishi M (1978) A neural map of auditory space in the owl. Science 200:795-797. CrossRef Medline

Lee CC, Sherman SM (2010) Topography and physiology of ascending streams in the auditory tectothalamic pathway. Proc Natl Acad Sci U S A 107:372-377. CrossRef Medline

Lee CC, Yanagawa Y, Imaizumi K (2015) Commissural functional topography of the inferior colliculus assessed in vitro. Hear Res 328:94-101. CrossRef Medline

Lein ES, Hawrylycz MJ, Ao N, Ayres M, Bensinger A, Bernard A, Boe AF, Boguski MS, Brockway KS, Byrnes EJ, Chen L, Chen L, Chen TM, Chin MC, Chong J, Crook BE, Czaplinska A, Dang CN, Datta S, Dee NR, et al. (2007) Genome-wide atlas of gene expression in the adult mouse brain. Nature 445:168-176. CrossRef Medline

Lesicko AM, Hristova TS, Maigler KC, Llano DA (2016) Connectional modularity of top-down and bottom-up multimodal inputs to the lateral cortex of the mouse inferior colliculus. J Neurosci 36:11037-11050. CrossRef Medline

Loftus WC, Bishop DC, Saint Marie RL, Oliver DL (2004) Organization of binaural excitatory and inhibitory inputs to the inferior colliculus from the superior olive. J Comp Neurol 472:330-344. CrossRef Medline

Loftus WC, Malmierca MS, Bishop DC, Oliver DL (2008) The cytoarchitecture of the inferior colliculus revisited: a common organization of the lateral cortex in rat and cat. Neuroscience 154:196-205. CrossRef Medline

Loftus WC, Bishop DC, Oliver DL (2010) Differential patterns of inputs create functional zones in central nucleus of inferior colliculus. J Neurosci 30:13396-13408. CrossRef Medline

Malmierca MS, Blackstad TW, Osen KK, Karagülle T, Molowny RL (1993) The central nucleus of the inferior colliculus in rat: a Golgi and computer reconstruction study of neuronal and laminar structure. J Comp Neurol 333:1-27. CrossRef Medline

Malmierca MS, Saint Marie RL, Merchán MA, Oliver DL (2005) Laminar inputs from dorsal cochlear nucleus and ventral cochlear nucleus to the central nucleus of the inferior colliculus: two patterns of convergence. Neuroscience 136:883-894. CrossRef Medline

Malmierca MS, Blackstad TW, Osen KK (2011) Computer-assisted 3-D reconstructions of Golgi-impregnated neurons in the cortical regions of the inferior colliculus of rat. Hear Res 274:13-26. CrossRef Medline

Menegas W, Bergan JF, Ogawa SK, Isogai Y, Umadevi Venkataraju K, Osten P, Uchida N, Watabe-Uchida M (2015) Dopamine neurons projecting to the posterior striatum form an anatomically distinct subclass. eLife 4:e10032. CrossRef Medline

Merchán M, Aguilar LA, Lopez-Poveda EA, Malmierca MS (2005) The inferior colliculus of the rat: quantitative immunocytochemical study of GABA and glycine. Neuroscience 136:907-925. CrossRef Medline

Nakamoto KT, Mellott JG, Killius J, Storey-Workley ME, Sowick CS, Schofield BR (2013a) Ultrastructural examination of the corticocollicular pathway in the guinea pig: a study using electron microscopy, neural tracers, and GABA immunocytochemistry. Front Neuroanat 7:13. CrossRef Medline

Nakamoto KT, Sowick CS, Schofield BR (2013b) Auditory cortical axons contact commissural cells throughout the guinea pig inferior colliculus. Hear Res 306:131-144. CrossRef Medline

Oliver D (2005) Neuronal organization in the inferior colliculus. In: The inferior colliculus (Winer JA, Schreiner CE, eds). New York: Springer.

Oliver DL, Morest DK (1984) The central nucleus of the inferior colliculus in the cat. J Comp Neurol 222:237-264. CrossRef Medline

Oliver DL, Kuwada S, Yin TC, Haberly LB, Henkel CK (1991) Dendritic and 
axonal morphology of HRP-injected neurons in the inferior colliculus of the cat. J Comp Neurol 303:75-100. CrossRef Medline

Oliver DL, Ostapoff EM, Beckius GE (1999) Direct innervation of identified tectothalamic neurons in the inferior colliculus by axons from the cochlear nucleus. Neuroscience 93:643-658. CrossRef Medline

Ono M, Bishop DC, Oliver DL (2017) Identified GABAergic and glutamatergic neurons in the mouse inferior colliculus share similar response properties. J Neurosci 37:8952-8964. CrossRef Medline

Patel MB, Sons S, Yudintsev G, Lesicko AM, Yang L, Taha GA, Pierce SM, Llano DA (2017) Anatomical characterization of subcortical descending projections to the inferior colliculus in mouse. J Comp Neurol 525:885900. CrossRef Medline

Paxinos G, Franklin KBJ (2001) The mouse brain in stereotaxic coordinates, Ed 2. San Diego: Academic.

Peruzzi D, Bartlett E, Smith PH, Oliver DL (1997) A monosynaptic GABAergic input from the inferior colliculus to the medial geniculate body in rat. J Neurosci 17:3766-3777. Medline

Robards MJ (1979) Somatic neurons in the brainstem and neocortex projecting to the external nucleus of the inferior colliculus: an anatomical study in the opossum. J Comp Neurol 184:547-565. CrossRef Medline

Saint Marie R, Baker R (1990) Neurotransmitter-specific uptake and retrograde transport of $[3 \mathrm{H}]$ glycine from the inferior colliculus by ipsilateral projections of the superior olivary complex and nuclei of the lateral lemniscus. Brain Res 524:244-253. CrossRef

Saldaña E, Feliciano M, Mugnaini E (1996) Distribution of descending projections from primary auditory neocortex to inferior colliculus mimics the topography of intracollicular projections. J Comp Neurol 371:15-40. CrossRef Medline

Saldaña E, Aparicio MA, Fuentes-Santamaría V, Berrebi AS (2009) Connections of the superior paraolivary nucleus of the rat: projections to the inferior colliculus. Neuroscience 163:372-387. CrossRef Medline

Shang C, Liu Z, Chen Z, Shi Y, Wang Q, Liu S, Li D, Cao P (2015) A parvalbumin-positive excitatory visual pathway to trigger fear responses in mice. Science 348:1472-1477. CrossRef Medline

Shen L, Zhao L, Hong B (2015) Frequency-specific adaptation and its underlying circuit model in the auditory midbrain. Front Neural Circuits 9:55. CrossRef Medline

Shneiderman A, Oliver DL, Henkel CK (1988) Connections of the dorsal nucleus of the lateral lemniscus: an inhibitory parallel pathway in the ascending auditory system? J Comp Neurol 276:188-208. CrossRef Medline

Syka J, Popelár J, Kvasnák E, Astl J (2000) Response properties of neurons in the central nucleus and external and dorsal cortices of the inferior colliculus in guinea pig. Exp Brain Res 133:254-266. CrossRef Medline

Tanaka I, Ezure K (2004) Overall distribution of GLYT2 mRNA-containing versus GAD67 mRNA-containing neurons and colocalization of both mRNAs in midbrain, pons, and cerebellum in rats. Neurosci Res 49:165178. CrossRef
Tokunaga A, Sugita S, Otani K (1984) Auditory and non-auditory subcortical afferents to the inferior colliculus in the rat. J Hirnforsch 25:461-472. Medline

Wall NR, De La Parra M, Callaway EM, Kreitzer AC (2013) Differential innervation of direct- and indirect-pathway striatal projection neurons. Neuron 79:347-360. CrossRef Medline

Watabe-Uchida M, Zhu L, Ogawa SK, Vamanrao A, Uchida N (2012) Whole-brain mapping of direct inputs to midbrain dopamine neurons. Neuron 74:858-873. CrossRef Medline

Wei P, Liu N, Zhang Z, Liu X, Tang Y, He X, Wu B, Zhou Z, Liu Y, Li J, Zhang Y, Zhou X, Xu L, Chen L, Bi G, Hu X, Xu F, Wang L (2015) Auditory cortex controls sound-driven innate defense behaviour through corticofugal projections to inferior colliculus. Nat Commun 6:6756. CrossRef Medline

Weissbourd B, Ren J, DeLoach KE, Guenthner CJ, Miyamichi K, Luo L (2014) Presynaptic partners of dorsal raphe serotonergic and GABAergic neurons. Neuron 83:645-662. CrossRef Medline

Whitley JM, Henkel CK (1984) Topographical organization of the inferior collicular projection and other connections of the ventral nucleus of the lateral lemniscus in the cat. J Comp Neurol 229:257-270. CrossRef Medline

Wickersham IR, Lyon DC, Barnard RJ, Mori T, Finke S, Conzelmann KK, Young JA, Callaway EM (2007) Monosynaptic restriction of transsynaptic tracing from single, genetically targeted neurons. Neuron 53:639_ 647. CrossRef Medline

Wilczynski W, Ryan MJ (2010) The behavioral neuroscience of anuran social signal processing. Curr Opin Neurobiol 20:754-763. CrossRef Medline

Willard FH, Martin GF (1983) The auditory brainstem nuclei and some of their projections to the inferior colliculus in the north American opossum. Neuroscience 10:1203-1232. CrossRef Medline

Winer JA, Schreiner CE (2005) The central auditory system: a functional analysis. In: The inferior colliculus (Winer JA, Schreiner CE, eds). New York: Springer.

Winer JA, Larue DT, Diehl JJ, Hefti BJ (1998) Auditory cortical projections to the cat inferior colliculus. J Comp Neurol 400:147-174. CrossRef Medline

Xiong XR, Liang F, Zingg B, Ji XY, Ibrahim LA, Tao HW, Zhang LI (2015) Auditory cortex controls sound-driven innate defense behaviour through corticofugal projections to inferior colliculus. Nat Commun 6:7224. CrossRef Medline

Zhang H, Kelly JB (2006) Responses of neurons in the rat's ventral nucleus of the lateral lemniscus to amplitude-modulated tones. J Neurophysiol 96:2905-2914. CrossRef Medline

Zhou J, Shore S (2006) Convergence of spinal trigeminal and cochlear nucleus projections in the inferior colliculus of the guinea pig. J Comp Neurol 495:100-112. CrossRef Medline 